\title{
Constraints on models of scalar and vector leptoquarks decaying to a quark and a neutrino at $\sqrt{s}=13 \mathrm{TeV}$
}

\author{
A. M. Sirunyan et al. \\ (CMS Collaboration)
}

(Received 25 May 2018; published 10 August 2018)

\begin{abstract}
The results of a previous search by the CMS Collaboration for squarks and gluinos are reinterpreted to constrain models of leptoquark (LQ) production. The search considers jets in association with a transverse momentum imbalance, using the $M_{\mathrm{T} 2}$ variable. The analysis uses proton-proton collision data at $\sqrt{s}=13 \mathrm{TeV}$, recorded with the CMS detector at the LHC in 2016 and corresponding to an integrated luminosity of $35.9 \mathrm{fb}^{-1}$. Leptoquark pair production is considered with LQ decays to a neutrino and a top, bottom, or light quark. This reinterpretation considers higher mass values than the original CMS search to constrain both scalar and vector LQs. Limits on the cross section for LQ pair production are derived at the 95\% confidence level depending on the LQ decay mode. A vector LQ decaying with a 50\% branching fraction to $t \nu$, and $50 \%$ to $b \tau$, has been proposed as part of an explanation of anomalous flavor physics results. In such a model, using only the decays to $t \nu$, LQ masses below $1530 \mathrm{GeV}$ are excluded assuming the Yang-Mills case with coupling $\kappa=1$, or $1115 \mathrm{GeV}$ in the minimal coupling case $\kappa=0$, placing the most stringent constraint to date from pair production of vector LQs.
\end{abstract}

DOI: 10.1103/PhysRevD.98.032005

\section{INTRODUCTION}

Leptoquarks (LQ) are hypothetical particles with quantum numbers of both quarks and leptons [1]. The spin of an LQ state is either 0 (scalar LQ, denoted LQS) or 1 (vector $\mathrm{LQ}$, denoted $\mathrm{LQ}_{\mathrm{V}}$ ). Leptoquarks appear in theories beyond the standard model (SM) such as grand unified theories [1-4], technicolor models [5-8], compositeness scenarios $[9,10]$, and $\mathrm{R}$ parity [11] violating supersymmetry (SUSY) [12-20].

A growing collection of anomalies have been observed in flavor physics by the $B A B A R$ [21,22], Belle [23-26], and LHCb [27-31] Collaborations. These have been explained as hints of lepton flavor universality violation in both charged- and neutral-current processes. Leptoquarks have been suggested as an explanation of these results [32-38]. In particular, the best fit model of Refs. [37,38] predicts an $\mathrm{LQ}_{\mathrm{V}}$ with a mass of $\mathcal{O}(\mathrm{TeV})$ decaying with $50 \%$ branching fraction to either a top quark and a neutrino $(t \nu)$ or a bottom quark and a tau lepton $(b \tau)$. Such a state would therefore be visible at the CERN LHC.

At the LHC, LQ can be produced either in pairs or singly in association with a lepton. In this paper, we focus on LQ

${ }^{*}$ Full author list given at the end of the article.

Published by the American Physical Society under the terms of the Creative Commons Attribution 4.0 International license. Further distribution of this work must maintain attribution to the author(s) and the published article's title, journal citation, and DOI. Funded by SCOAP . pair production with both decaying to a neutrino and a top, bottom, or light quark (any single one of up, down, strange, or charm). The dominant leading-order (LO) diagrams for pair production at the LHC are shown in Fig. 1. The models for $\mathrm{LQ}_{S}$ and $\mathrm{LQ}_{\mathrm{V}}$ pair production are taken from Ref. [38], which provides a concrete implementation of the models from Ref. [37]. For $\mathrm{LQ}_{S}$, the pair production cross section depends only on the $\mathrm{LQ}$ mass. For $\mathrm{LQ}_{\mathrm{V}}$, there are additional constraints imposed by unitarity at high energy scales leading to model dependent solutions and thus production cross sections. In the model developed to explain the flavor physics anomalies [38], the additional relevant parameter for the $\mathrm{LQ}_{\mathrm{V}}$ pair production cross section is $\kappa$, a dimensionless coupling that is 1 in the Yang-Mills case and 0 in the minimal coupling case, and we consider both values.
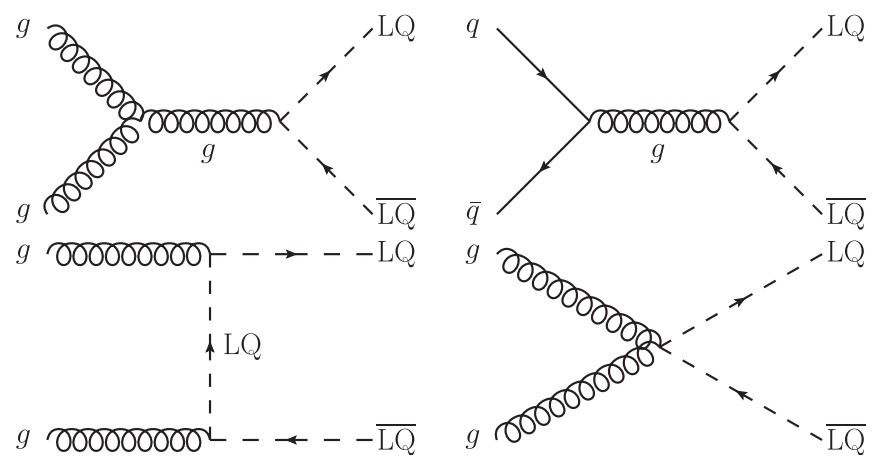

FIG. 1. Dominant LO diagrams for LQ pair production in proton-proton collisions. 
For $\kappa=1$, the cross section for $\mathrm{LQ}_{\mathrm{V}}$ pair production is a factor of 5-20 times larger than that of $\mathrm{LQ}_{\mathrm{S}}$, depending on the LQ mass. The other free parameters in the $\mathrm{LQ}_{\mathrm{V}}$ model are $g_{t_{\mathrm{L}}}$ and $g_{b_{\mathrm{L}}}$, the couplings of the $\mathrm{LQ}_{\mathrm{V}}$ to $t \nu$ and $b \tau$ pairs respectively, but they do not affect the cross section or kinematics for pair production.

The pair production of $\mathrm{LQ}_{\mathrm{S}}$, each decaying to a quark and neutrino, results in the same final states and kinematics as those considered in searches for squark pair production in R-parity conserving SUSY, assuming that the squark decays directly to a quark and a massless neutralino [39]. In both cases, the initial particles are scalars $\left(\mathrm{LQ}_{\mathrm{S}}\right.$ or squark) produced strongly via quantum chromodynamics (QCD), and the decay products are a quark and a nearly massless fermion (neutrino or neutralino). In practice, the decay products in $\mathrm{LQ}_{\mathrm{V}}$ pair production are also found to have similar kinematics [39]. Searches for squark pair production are therefore already optimized to search for LQ pair production. Constraints on LQ production with decays to a quark and a neutrino have been placed using LHC data by the ATLAS [40] and CMS [41-43] Collaborations, either by reinterpreting existing squark searches, or considering mixed branching fraction scenarios with an LQ also decaying to a quark and a charged lepton. Searches at the Tevatron covering the same signatures have been performed by the CDF [44-46] and D0 [47-49] Collaborations. Direct searches for single LQ production have also been performed at HERA by the H1 [50] and ZEUS [51] Collaborations, placing constraints that are most stringent for an LQ with large coupling to an electron and a quark, and large branching fraction for the decay to a quark and a neutrino. Searches have also been performed by the ATLAS [52], CMS [53], CDF [54,55], and D0 [56] Collaborations for an LQ decaying to the $b \tau$ channel as predicted in the model of Refs. [37,38].

The results from the CMS search for jets in association with a transverse momentum imbalance $\left(p_{\mathrm{T}}^{\text {miss }}\right)$ using the $M_{\mathrm{T} 2}$ variable [57], reported in Ref. [58] and initially interpreted for squark and gluino production, have recently been reinterpreted as part of a review of LQ searches to place the strongest limits on the pair production of LQ decaying to a quark and a neutrino [39]. However, for $L Q_{V}$, the pair production cross sections are large enough that the mass range of interest was not covered by the simulated samples used in Ref. [58]. In particular, for an $\mathrm{LQ}_{\mathrm{V}}$ decaying to $t \nu$ as predicted to explain the flavor physics anomalies, the mass limit was derived from a flat extrapolation assuming that the cross section limit stayed the same at higher masses. To improve upon these constraints, in this paper we present an extended interpretation of the search from Ref. [58], where the selections, predictions, and uncertainties of the original analysis have not been changed. Exploiting the similarity in final states between squark and LQ pair production, we verify that the acceptance of our analysis is consistent within uncertainties for squark, $\mathrm{LQ}_{\mathrm{S}}$, and $\mathrm{LQ}_{\mathrm{V}}$ pair production for the same squark/ LQ mass, assuming a neutralino mass of $1 \mathrm{GeV}$ in the squark case. We thus proceed to use simulated squark samples to place limits on both $\mathrm{LQ}_{\mathrm{S}}$ and $\mathrm{LQ}_{\mathrm{V}}$ production. Using the full analysis information including all signal regions and correlations, we extend the interpretations from Ref. [58] to higher mass values, allowing us to improve the upper limits on LQ pair production cross sections in the $t \nu$ decay channel by as much as a factor of 2.8 over the flat extrapolation assumed in Ref. [39]. With this approach, we derive the strongest coupling-independent constraints to date on the anomaly-inspired model of Refs. [37,38].

\section{ANALYSIS OVERVIEW}

This study reinterprets the CMS search for jets and $p_{\mathrm{T}}^{\text {miss }}$ using the $M_{\mathrm{T} 2}$ variable. The analysis is unchanged with respect to Ref. [58], where a full description can be found, and is briefly summarized here. The search uses protonproton collision data at $\sqrt{s}=13 \mathrm{TeV}$, recorded with the CMS detector in 2016, and corresponding to an integrated luminosity of $35.9 \mathrm{fb}^{-1}$. A description of the CMS detector, together with a definition of the coordinate system used and the relevant kinematic variables, can be found in Ref. [59].

Event reconstruction is based on the particle-flow (PF) algorithm [60]. Jets are clustered from PF candidates using the anti- $k_{\mathrm{T}}$ clustering algorithm [61] with a distance parameter of $\mathrm{R}=0.4$, as implemented in the FASTJET package [62], and are required to have pseudorapidity $|\eta|<2$.4. Jets with transverse momentum $p_{\mathrm{T}}>20 \mathrm{GeV}$ are identified as originating from $b$ quarks (" $b$ tagged") using the combined secondary vertex algorithm [63], and the number of $b$-tagged jets is denoted $N_{b}$. For all other quantities considered in the analysis, jets are required to satisfy $p_{\mathrm{T}}>30 \mathrm{GeV}$. The number of passing jets is denoted $N_{\mathrm{j}}$, and the variable $H_{\mathrm{T}}$ is defined as the scalar sum of jet $p_{\mathrm{T}}$. The missing transverse momentum vector, $\vec{p}_{\mathrm{T}}^{\text {miss }}$, is defined as the negative vector sum of the momenta of all reconstructed PF candidates originating from the primary vertex, projected onto the plane perpendicular to the proton beams. Its magnitude is referred to as $p_{\mathrm{T}}^{\text {miss }}$.

At the trigger level, events are selected by requiring large $H_{\mathrm{T}}$, jet $p_{\mathrm{T}}$, or $p_{\mathrm{T}}^{\text {miss }}$. The trigger selections have efficiency greater than $98 \%$ for events with offline reconstructed values of $p_{\mathrm{T}}^{\text {miss }}>250 \mathrm{GeV}$ or $H_{\mathrm{T}}>1000 \mathrm{GeV}$. The baseline selection requires $N_{\mathrm{j}} \geq 1$, and events must pass either $p_{\mathrm{T}}^{\text {miss }}>30 \mathrm{GeV}$ if they have $H_{\mathrm{T}}>1000 \mathrm{GeV}$, or $p_{\mathrm{T}}^{\text {miss }}>$ $250 \mathrm{GeV}$ if they have $250<H_{\mathrm{T}}<1000 \mathrm{GeV}$. Further baseline requirements include that $\vec{p}_{\mathrm{T}}^{\text {miss }}$ is not aligned in the azimuthal angle $\phi$ with any of the four leading jets in $p_{\mathrm{T}}$, that the negative vector sum of jet transverse momenta, $\vec{H}_{\mathrm{T}}^{\text {miss }}$, is consistent with $\vec{p}_{\mathrm{T}}^{\text {miss }}$, and that no loosely identified charged leptons or isolated tracks are found in the event. For events with $N_{\mathrm{j}} \geq 2$, the variable $M_{\mathrm{T} 2}$ is computed from the jets and the $\vec{p}_{\mathrm{T}}^{\text {miss }}$ as described in Ref. [58]. The $M_{\mathrm{T} 2}$ 

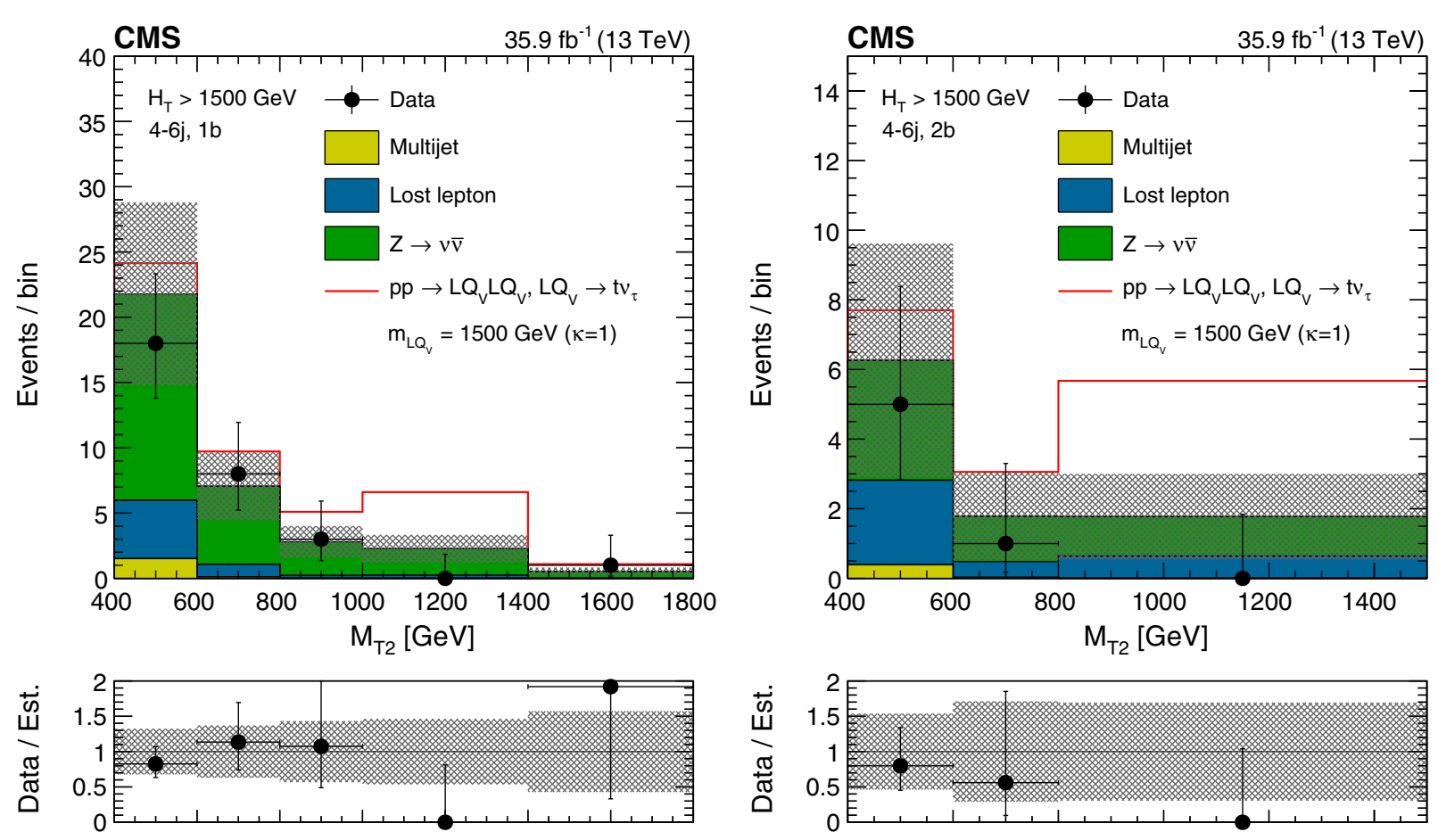

FIG. 2. Distributions of $M_{\mathrm{T} 2}$ showing data, the background predictions, and a hypothetical LQ signal with LQ mass of $1500 \mathrm{GeV}$ decaying with $100 \%$ branching fraction to $t \nu_{\tau}$. The cross section used for the LQ $Q_{\mathrm{V}}$ signal assumes $\kappa=1$, and the signal is stacked on top of the background predictions. The black points show the observed data, with the statistical uncertainties represented by the vertical bars, and the bin widths represented by the horizontal bars. The rightmost bin in each plot also includes events with larger values of $M_{\mathrm{T} 2}$. The hatched band shows the uncertainty in the background prediction including both statistical and systematic components. The lower pane of each plot shows the ratio of observed data over predicted background. The categories require $H_{\mathrm{T}}>1500 \mathrm{GeV}, 4 \leq N_{\mathrm{j}} \leq 6$, and (left) $N_{b}=1$ or (right) $N_{b}=2$.

variable takes on small values for events where the momentum imbalance arises from jet mismeasurement, typical of the QCD multijet background, and it yields larger values in events with genuine $\vec{p}_{\mathrm{T}}^{\text {miss }}$. The baseline selection for events with $N_{\mathrm{j}} \geq 2$ requires $M_{\mathrm{T} 2}>200 \mathrm{GeV}$, which is raised to $M_{\mathrm{T} 2}>400 \mathrm{GeV}$ for events with $H_{\mathrm{T}}>1500 \mathrm{GeV}$ to further reject multijet background.

Events with $N_{\mathrm{j}} \geq 2$ passing the baseline selection are categorized according to four variables: $H_{\mathrm{T}}, M_{\mathrm{T} 2}, N_{\mathrm{j}}$, and $N_{b}$. Events with $N_{\mathrm{j}}=1$ are categorized according to the jet $p_{\mathrm{T}}$ and the presence or absence of a $b$-tagged jet. The analysis spans a wide range of kinematics and jet multiplicities, containing 213 search bins in total, to maintain sensitivity to a variety of new physics signatures.

The SM backgrounds to the search comprise three classes of processes: $Z+$ jets production with the decay $Z \rightarrow \nu \bar{\nu}, W+$ jets or $t \bar{t}+$ jets production with the decay $W \rightarrow \ell \nu$ where the charged lepton is outside the acceptance or not identified ("lost lepton"), and QCD multijet production where $\vec{p}_{\mathrm{T}}^{\text {miss }}$ arises from jet mismeasurement. Each of these backgrounds is predicted primarily from data control regions: $Z+$ jets from $Z \rightarrow \ell^{+} \ell^{-}$events, $W+$ jets and $t \bar{t}+$ jets from events containing an identified electron or muon, and QCD multijets from events where at least one of the jets is aligned in $\phi$ with $\vec{p}_{\mathrm{T}}^{\text {miss }}$.
Depending on the LQ mass and decay products, different search bins provide the greatest signal sensitivity. Figure 2 shows the $M_{\mathrm{T} 2}$ distribution for data, the background predictions, and a hypothetical $\mathrm{LQ}_{\mathrm{V}}$ signal in the two most sensitive search categories for an LQ of mass $1500 \mathrm{GeV}$ decaying with $100 \%$ branching fraction to $t \nu$.

Taking into account all of the analysis bins, no significant deviations from the SM prediction are observed. Simultaneous maximum likelihood fits to data yields in all bins are performed, and the results are interpreted as limits on the production cross sections of hypothetical scenarios of LQ pair production.

\section{SIMULATED SAMPLES}

Monte Carlo (MC) simulated samples are used to estimate the background from some SM processes, to assess systematic uncertainties in prediction methods that rely on data, and to calculate the selection efficiency for signal models. The main background samples $(Z+$ jets, $W+$ jets, and $t \bar{t}+$ jets), as well as signal samples, are generated at LO precision in perturbative QCD with the MADGRAPH5_AMC@NLO v2.3.3 generator [64]. Up to four, three, or two additional partons are considered in the matrix element calculations for the generation of the $V+$ jets $(V=Z, W), t \bar{t}+$ jets, and signal samples, 
respectively. The NNPDF3.0 LO [65] parton distribution functions (PDFs) are used in the event generation. Parton showering and fragmentation are performed using the PYTHIA v8.212 [66] generator and the CUETP8M1 tune [67]. The potential double counting of the partons generated with MADGRAPH5_AMC@NLO and those with PYTHIA is removed using the MLM [68] matching scheme. The samples used for the SM backgrounds are unchanged from Ref. [58], and the details of the sample generation for other SM processes are described further there.

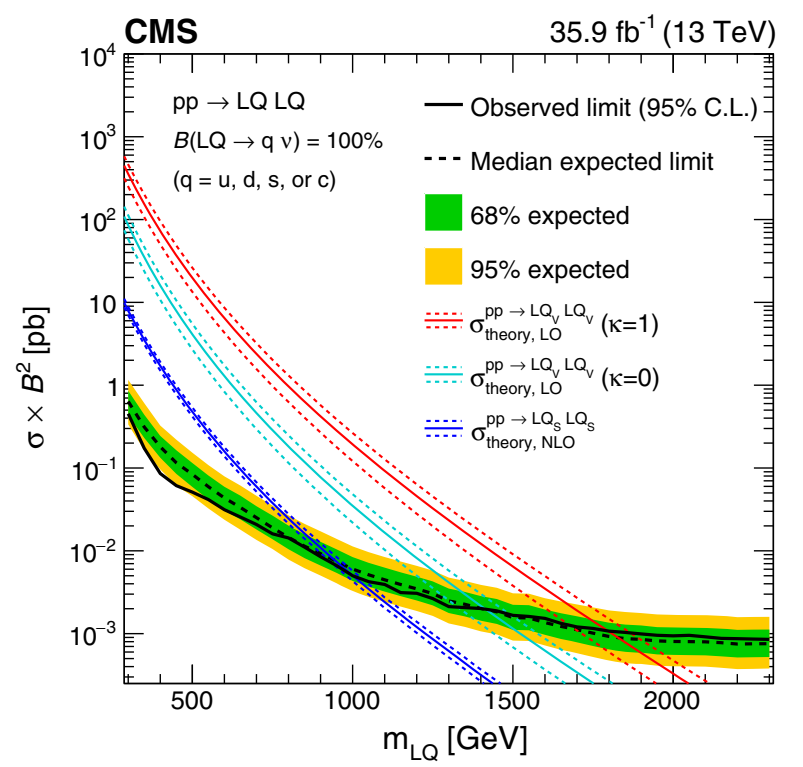

Additional proton-proton interactions in the same or nearby bunch crossings (pileup) are generated with PYTHIA and superimposed on the hard collisions. The response of the CMS detector to SM background samples is simulated using a GEANT4-based model [69], while that to new physics signals is modeled using the CMS fast simulation package [70]. All simulated events are processed with the same chain of reconstruction programs as used for collision data. Corrections are applied to simulated samples to account for differences between measurements in data

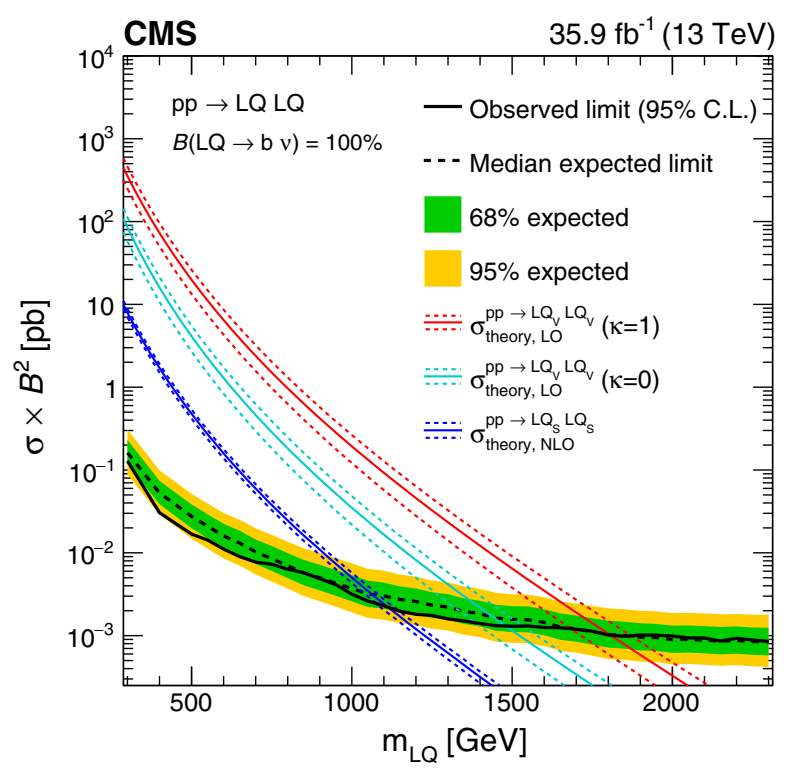

$35.9 \mathrm{fb}^{-1}(13 \mathrm{TeV})$

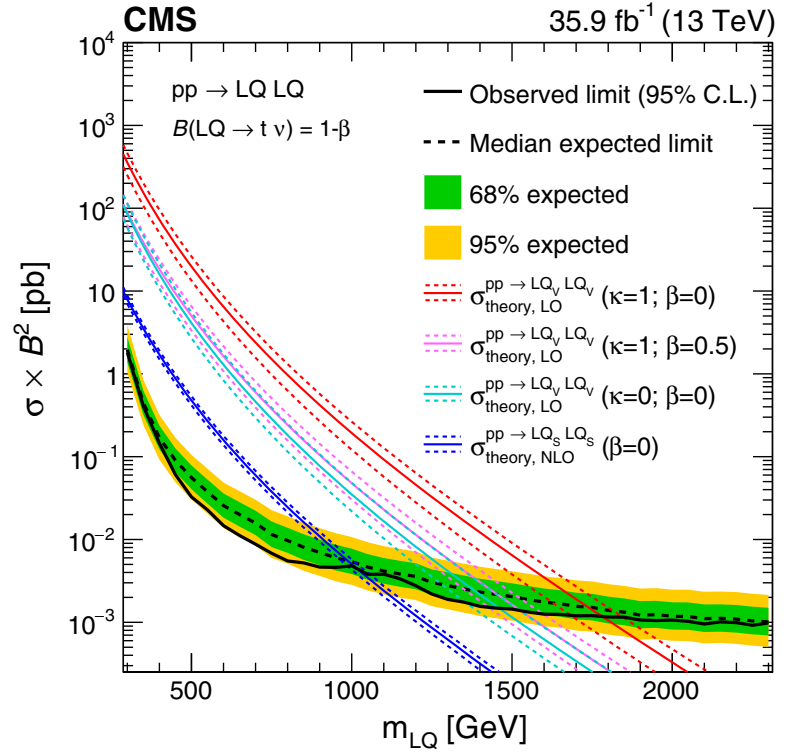

FIG. 3. The $95 \%$ C.L. upper limits on the production cross sections as a function of LQ mass for LQ pair production decaying with $100 \%$ branching fraction to a neutrino and (upper left) a light quark (one of $u, d, s$, or $c$ ), (upper right) a bottom quark, or (lower) a top quark. The solid (dashed) black line represents the observed (median expected) exclusion. The inner green (outer yellow) band indicates the region containing $68(95 \%)$ of the distribution of limits expected under the background-only hypothesis. The dark blue lines show the theoretical cross section for $L_{S}$ pair production with its uncertainty. The red (light blue) lines show the same for $L Q_{V}$ pair production assuming $\kappa=1(0)$. Also shown (lower) in magenta is the product of the theoretical cross section and the square of the branching fraction $(B)$, for vector LQ pair production assuming $\kappa=1$ and a $50 \%$ branching fraction to $t \nu_{\tau}$, with the remaining $50 \%$ to $b \tau$. 
and the GEANT4 simulation of the trigger, $b$ tagging, and lepton selection efficiencies. Additional differences arising from the fast simulation modeling of selection efficiencies, as well as from the modeling of $p_{\mathrm{T}}^{\text {miss }}$, are corrected in the fast simulation and included in the systematic uncertainties considered.

The generated signal samples used for this interpretation consist of simplified models [71-75] of squark pair production, with the squark decaying to a quark of the same flavor and a neutralino with mass of $1 \mathrm{GeV}$. Three samples are generated with different squark flavors: "light" squarks with an equal fraction of $(\tilde{u}, \tilde{d}, \tilde{s}, \tilde{c})$, bottom squarks, and top squarks. Squark masses up to $2300 \mathrm{GeV}$ are generated, compared to Ref. [58] where the generated samples extended to masses of $1800 \mathrm{GeV}$ for light squarks, $1450 \mathrm{GeV}$ for bottom squarks, and $1200 \mathrm{GeV}$ for top squarks. Below those mass values, the previous samples generated with the same configuration are used.

Samples of pair production of $\mathrm{LQ}_{\mathrm{S}}$ and $\mathrm{LQ}_{\mathrm{V}}$ are also generated for a limited number of $L Q$ mass values, to verify that the acceptance of the analysis at generator level is consistent with the squark samples used. Samples of $L_{S}$ pair production are generated with the PYTHIA v8.205 generator, using the NNPDF2.3 LO [76] PDFs. Samples of $\mathrm{LQ}_{\mathrm{V}}$ pair production are generated with the MADGRAPH5_AMC@NLO generator at LO precision in perturbative QCD, including up to two additional partons in the matrix element calculations and using the MLM matching scheme and NNPDF3.1 LO [77] PDFs. The variables defined in Sec. II are computed at generator level, and the kinematics of the generated squark samples are compared to those of $\mathrm{LQ}_{\mathrm{S}}$ and $\mathrm{LQ}_{\mathrm{V}}$ pair production samples. The acceptance of both the baseline analysis selection and the kinematic requirements for the most sensitive signal regions is found to be consistent within statistical uncertainties of $\sim 3 \%-10 \%$ for the squark, $L Q_{S}$, and $\mathrm{LQ}_{\mathrm{V}}$ samples. The statistical uncertainty of the simulated signal samples is included when using the squark samples to set limits on LQ pair production, and based on this study no additional correction for, or systematic uncertainty in, the signal acceptance is applied.

To improve the modeling of the multiplicity of additional jets beyond those from the hard scatter process, we weight the signal MC events based on the number of such jets, denoted $N_{\mathrm{j}}^{\mathrm{ISR}}$ for initial-state radiation (ISR). The weighting factors are derived from a control region enriched in $t \bar{t}$ events, obtained by selecting events with exactly two leptons $(e e, \mu \mu$ or $e \mu$ ) and exactly two $b$-tagged jets. The factors are chosen to make the simulated jet multiplicity agree with data, and they vary between 0.92 for $N_{\mathrm{j}}^{\mathrm{ISR}}=1$ and 0.51 for $N_{\mathrm{j}}^{\mathrm{ISR}} \geq 6$. We take one half of the deviation from unity as the systematic uncertainty in these reweighting factors, as an estimate of the differences between $t \bar{t}$ and signal production.

The cross sections for $\mathrm{LQ}_{\mathrm{S}}$ or $\mathrm{LQ}_{\mathrm{V}}$ pair production are computed to next-to-leading-order (NLO) or LO precision in perturbative QCD, following Ref. [38] and using the NNPDF2.3 NLO or LO PDF set, respectively. In the LQV model, we assume $g_{t_{\mathrm{L}}}=g_{b_{\mathrm{L}}}=0.1$, and either $\kappa=1$ or $\kappa=0$, as predicted to explain the flavor physics anomalies. The uncertainties in cross section calculations arise from PDF variations and from the renormalization and factorization scale variations. For PDF uncertainties, the NNPDF2.3 PDF set variations are used. For scale uncertainties, renormalization and factorization scales are varied up and down by a factor of two with respect to the nominal values. The theoretical uncertainties in the cross section are not included in the limit calculation but displayed separately in Fig. 3.

\section{INTERPRETATION}

The search results of Ref. [58] are interpreted to place cross section limits on LQ pair production as a function of the LQ mass. A modified frequentist approach is used, employing the $\mathrm{CL}_{\mathrm{s}}$ criterion and an asymptotic formulation [78-81]. The uncertainties in the signal acceptance and efficiency, and in the background predictions, are incorporated as nuisance parameters. The observed data yields in control regions are parameterized using gamma functions, while other nuisance parameters are implemented using log-normal functions, whose widths reflect the size of the systematic uncertainty.

The following sources of uncertainty in the signal acceptance and efficiency are evaluated and taken to be fully correlated across all analysis bins: determination of the integrated luminosity [82], trigger efficiency, lepton identification and isolation efficiency, lepton efficiency modeling in fast simulation, $b$ tagging efficiency, jet energy scale, modeling of $p_{\mathrm{T}}^{\text {miss }}$ in fast simulation, modeling of ISR, simulation of pileup, and variations of the generator factorization and renormalization scales. The statistical uncertainty of the simulated signal samples is taken to be uncorrelated in every bin. The total uncertainty in the signal acceptance is typically around 5\%-25\% in the most sensitive analysis bins. A detailed discussion of the uncertainties in the background prediction can be found in Ref. [58].

Exclusion limits at the 95\% confidence level (C.L.) on the cross section of LQ pair production are shown in Fig. 3. In each case, we assume that there is only one LQ state with low enough mass to be produced at the LHC, and that any other potential LQ states have masses too large to be produced. We assume that the LQ decays with $100 \%$ branching fraction to a neutrino and a single type of quark, as specified below. In the simulated samples used to determine the signal acceptance, and for the cross sections displayed, we consider only LQ pair production and not single LQ production.

We first consider LQ decays to a neutrino and a light quark, which can be any single one of the $u, d, s$, or $c$ quarks. As the analysis includes categorization in the 
TABLE I. Summary of the observed (expected) mass limits at the 95\% C.L., and the cross sections $\sigma$ that correspond to the excluded mass values. The columns show scalar or vector leptoquarks with the choice of $\kappa$, while the rows show the LQ decay channel.

\begin{tabular}{|c|c|c|c|c|c|c|}
\hline & \multicolumn{2}{|c|}{$\mathrm{LQ}_{\mathrm{S}}$} & \multicolumn{2}{|c|}{$\mathrm{LQ}_{\mathrm{V}}, \kappa=1$} & \multicolumn{2}{|c|}{$\mathrm{LQ}_{\mathrm{V}}, \kappa=0$} \\
\hline & Mass [GeV] & $\sigma[\mathrm{fb}]$ & Mass [GeV] & $\sigma[\mathrm{fb}]$ & Mass [GeV] & $\sigma[\mathrm{fb}]$ \\
\hline $\begin{array}{l}\mathrm{LQ} \rightarrow q \nu \\
q=u, d, s, \text { or } c\end{array}$ & $980(940)$ & $5.9(8.0)$ & $1790(1830)$ & $1.1(0.9)$ & $1410(1415)$ & $2.0(2.0)$ \\
\hline $\mathrm{LQ} \rightarrow b \nu$ & 1100 (1070) & $2.4(3.0)$ & $1810(1800)$ & $1.0(1.1)$ & 1475 (1440) & $1.3(1.7)$ \\
\hline $\mathrm{LQ} \rightarrow t \nu$ & $1020(980)$ & $4.3(5.9)$ & $1780(1740)$ & $1.2(1.5)$ & $1460(1385)$ & $1.5(2.4)$ \\
\hline $\mathrm{LQ} \rightarrow\left\{\begin{array}{l}t \nu(B=50 \%) \\
b \tau(B=50 \%)\end{array}\right.$ & $\ldots$ & $\ldots$ & $1530(1460)$ & $1.3(2.1)$ & 1115 (1095) & $3.7(4.2)$ \\
\hline
\end{tabular}

number of $b$-tagged jets, and the probability for a $c$ quark to pass the $b$ tagging selection is larger than that of the $u, d$, and $s$ quarks, we check whether the cross section limit obtained for an LQ decaying to $c \nu$ differs significantly from an LQ decaying to a neutrino and one of the other light quarks. The cross section limit differs by at most $10 \%$, resulting in a negligible impact on the mass limit, and we therefore do not produce separate limit results for these cases. The observed and expected limits on the LQ mass, and the corresponding cross sections for the excluded mass values, are summarized in Table I.

The observed limit is more stringent than expected by up to 2 standard deviations in the LQ mass range of about 400-600 GeV for a decay to a light or bottom quark and a neutrino, and in the range of about 500-900 GeV for the $t \nu$ decay channel. The most sensitive analysis bins differ in each case, primarily in the $N_{\mathrm{j}}$ and $N_{b}$ requirements. The background estimates for these bins are derived from statistically independent control regions, so the predictions and uncertainties are largely uncorrelated among these interpretations.

The model proposed in Refs. [37,38] as an explanation of the flavor physics anomalies predicts an $\mathrm{LQ}_{\mathrm{V}}$ with $50 \%$ branching fraction to each of the $t \nu$ and $b \tau$ channels. As our analysis removes events with charged leptons, including hadronically decaying $\tau$ leptons, we only consider the $25 \%$ of events where both LQ decay to $t \nu$ to place constraints on this model. We show the theoretical prediction for this branching fraction as a separate curve in Fig. 3 (lower) assuming $\kappa=1$. For $\kappa=1$, we find an observed (expected) limit on the $\mathrm{LQ}_{\mathrm{V}}$ mass of 1530 (1460) GeV, while for $\kappa=0$ we obtain a limit of 1115 (1095) GeV. The mass limit in the $\kappa=1$ case corresponds to a value of $1.3(2.1) \mathrm{fb}$ for the product of the LQ pair production cross section and the square of the branching fraction, while for $\kappa=0$, the value is 3.7 (4.2) fb.

\section{SUMMARY}

The CMS search for jets and missing transverse momentum using the $M_{\mathrm{T} 2}$ variable has been reinterpreted to place limits on leptoquark (LQ) pair production, where the LQ decays with $100 \%$ branching fraction to a quark and a neutrino. The search uses proton-proton collision data at $\sqrt{s}=13 \mathrm{TeV}$, recorded with the CMS detector in 2016 and corresponding to an integrated luminosity of $35.9 \mathrm{fb}^{-1}$. Leptoquark decays to a neutrino and a top, bottom, or light quark are considered. Compared to the original result, higher masses are considered to place exclusion limits on scalar LQs, and on vector LQs assuming either the YangMills $(\kappa=1)$ or minimal $(\kappa=0)$ coupling scenarios. Assuming that there is only one LQ state within mass reach of the LHC and that it decays to a light quark and a neutrino, masses below 980, 1790, and $1410 \mathrm{GeV}$ are excluded at the $95 \%$ confidence level by the observed data in the scalar, vector $\kappa=1$, and vector $\kappa=0$ scenarios. For an LQ decaying to $b \nu$, masses below 1100, 1810, and $1475 \mathrm{GeV}$ are excluded, while for an LQ decaying to $t \nu$, masses below 1020, 1780, and $1460 \mathrm{GeV}$ are excluded. In the model of Refs. [37,38], a vector LQ with $50 \%$ branching fraction to $t \nu$, and $50 \%$ to $b \tau$, is predicted to explain anomalous flavor physics results. Masses below 1530 (1115) GeV are excluded for such a state assuming $\kappa=1(\kappa=0)$, considering only the events with both LQ decaying to $t \nu$, providing the strongest constraint to date in this model from pair production.

\section{ACKNOWLEDGMENTS}

We congratulate our colleagues in the CERN accelerator departments for the excellent performance of the LHC and thank the technical and administrative staffs at CERN and at other CMS institutes for their contributions to the success of the CMS effort. In addition, we gratefully acknowledge the computing centers and personnel of the Worldwide LHC Computing Grid for delivering so effectively the computing infrastructure essential to our analyses. Finally, we acknowledge the enduring support for the construction and operation of the LHC and the CMS detector provided by the following funding agencies: BMWFW and FWF (Austria); FNRS and FWO (Belgium); CNPq, CAPES, FAPERJ, and FAPESP (Brazil); MES (Bulgaria); CERN; CAS, MoST, and NSFC (China); COLCIENCIAS (Colombia); MSES and CSF (Croatia); RPF (Cyprus); 
SENESCYT (Ecuador); MoER, ERC IUT, and ERDF (Estonia); Academy of Finland, MEC, and HIP (Finland); CEA and CNRS/IN2P3 (France); BMBF, DFG, and HGF (Germany); GSRT (Greece); NKFIA (Hungary); DAE and DST (India); IPM (Iran); SFI (Ireland); INFN (Italy); MSIP and NRF (Republic of Korea); LAS (Lithuania); MOE and UM (Malaysia); BUAP, CINVESTAV, CONACYT, LNS, SEP, and UASLP-FAI (Mexico); MBIE (New Zealand); PAEC (Pakistan); MSHE and NSC (Poland); FCT (Portugal); JINR (Dubna); MON, RosAtom, RAS and RFBR (Russia); MESTD (Serbia); SEIDI, CPAN, PCTI and FEDER (Spain); Swiss Funding Agencies (Switzerland); MST (Taipei); ThEPCenter, IPST, STAR, and NSTDA (Thailand); TUBITAK and TAEK (Turkey); NASU and SFFR (Ukraine); STFC (United Kingdom); DOE and NSF (USA). Individuals have received support from the Marie-Curie program and the European Research Council and Horizon 2020 Grant, contract No. 675440 (European Union); the Leventis Foundation; the A. P. Sloan Foundation; the Alexander von Humboldt Foundation; the Belgian Federal Science Policy Office; the Fonds pour la Formation à la Recherche dans l'Industrie et dans l'Agriculture (FRIA-Belgium); the Agentschap voor Innovatie door Wetenschap en Technologie (IWTBelgium); the F.R.S.-FNRS and FWO (Belgium) under the "Excellence of Science-EOS"-be.h Project
No. 30820817; the Ministry of Education, Youth and Sports (MEYS) of the Czech Republic; the Lendület ("Momentum") Program and the János Bolyai Research Scholarship of the Hungarian Academy of Sciences, the New National Excellence Program ÚNKP, the NKFIA research Grants Nos. 123842, 123959, 124845, 124850 and 125105 (Hungary); the Council of Science and Industrial Research, India; the HOMING PLUS program of the Foundation for Polish Science, cofinanced from European Union, Regional Development Fund, the Mobility Plus program of the Ministry of Science and Higher Education, the National Science Center (Poland), contracts Harmonia 2014/14/M/ST2/00428, Opus 2014/13/ B/ST2/02543, 2014/15/B/ST2/03998, and 2015/19/B/ST2/ 02861, Sonata-bis 2012/07/E/ST2/01406; the National Priorities Research Program by Qatar National Research Fund; the Programa Estatal de Fomento de la Investigación Científica y Técnica de Excelencia María de Maeztu, Grant No. MDM-2015-0509 and the Programa Severo Ochoa del Principado de Asturias; the Thalis and Aristeia programs cofinanced by EU-ESF and the Greek NSRF; the Rachadapisek Sompot Fund for Postdoctoral Fellowship, Chulalongkorn University and the Chulalongkorn Academic into Its 2nd Century Project Advancement Project (Thailand); the Welch Foundation, Contract No. C-1845; and the Weston Havens Foundation (USA).
[1] J. C. Pati and A. Salam, Unified lepton-hadron symmetry and a gauge theory of the basic interactions, Phys. Rev. D 8, 1240 (1973).

[2] J. C. Pati and A. Salam, Lepton number as the fourth color, Phys. Rev. D 10, 275 (1974); Erratum, Phys. Rev. D11, 703(E) (1975).

[3] H. Georgi and S. L. Glashow, Unity of All Elementary Particle Forces, Phys. Rev. Lett. 32, 438 (1974).

[4] H. Fritzsch and P. Minkowski, Unified interactions of leptons and hadrons, Ann. Phys. (N.Y.) 93, 193 (1975).

[5] S. Dimopoulos and L. Susskind, Mass without scalars, Nucl. Phys. B155, 237 (1979).

[6] S. Dimopoulos, Technicoloured signatures, Nucl. Phys. B168, 69 (1980).

[7] E. Farhi and L. Susskind, Technicolour, Phys. Rep. 74, 277 (1981).

[8] K. D. Lane and M. V. Ramana, Walking technicolor signatures at hadron colliders, Phys. Rev. D 44, 2678 (1991).

[9] B. Schrempp and F. Schrempp, Light leptoquarks, Phys. Lett. 153B, 101 (1985).

[10] B. Gripaios, Composite leptoquarks at the LHC, J. High Energy Phys. 02 (2010) 045.

[11] G. R. Farrar and P. Fayet, Phenomenology of the production, decay, and detection of new hadronic states associated with supersymmetry, Phys. Lett. 76B, 575 (1978).
[12] P. Ramond, Dual theory for free fermions, Phys. Rev. D 3, 2415 (1971).

[13] Yu. A. Golfand and E. P. Likhtman, Extension of the algebra of Poincaré group generators and violation of $\mathrm{p}$ invariance, JETP Lett. 13, 323 (1971).

[14] A. Neveu and J. H. Schwarz, Factorizable dual model of pions, Nucl. Phys. B31, 86 (1971).

[15] D. V. Volkov and V. P. Akulov, Possible universal neutrino interaction, JETP Lett. 16, 438 (1972).

[16] J. Wess and B. Zumino, A Lagrangian model invariant under supergauge transformations, Phys. Lett. 49B, 52 (1974).

[17] J. Wess and B. Zumino, Supergauge transformations in four dimensions, Nucl. Phys. B70, 39 (1974).

[18] P. Fayet, Supergauge invariant extension of the Higgs mechanism and a model for the electron and its neutrino, Nucl. Phys. B90, 104 (1975).

[19] H. P. Nilles, Supersymmetry, supergravity and particle physics, Phys. Rep. 110, 1 (1984).

[20] R. Barbier, C. Bérat, M. Besançon, M. Chemtob, A. Deandrea, E. Dudas, P. Fayet, S. Lavignac, G. Moreau, E. Perez, and Y. Sirois, R-parity violating supersymmetry, Phys. Rep. 420, 1 (2005).

[21] J.P. Lees et al. (BABAR Collaboration), Evidence for an Excess of $\bar{B} \rightarrow D^{(*)} \tau^{-} \bar{\nu}_{\tau}$ Decays, Phys. Rev. Lett. 109, 101802 (2012). 
[22] J. P. Lees et al. (BABAR Collaboration), Measurement of an excess of $\bar{B} \rightarrow D^{(*)} \tau^{-} \bar{\nu}_{\tau}$ decays and implications for charged Higgs bosons, Phys. Rev. D 88, 072012 (2013).

[23] A. Matyja et al. (Belle Collaboration), Observation of $B^{0} \rightarrow D^{*-} \tau^{+} \nu_{\tau}$ Decay at Belle, Phys. Rev. Lett. 99, 191807 (2007).

[24] A. Bozek et al. (Belle Collaboration), Observation of $B^{+} \rightarrow \bar{D}^{* 0} \tau^{+} \nu_{\tau}$ and evidence for $B^{+} \rightarrow \bar{D}^{0} \tau^{+} \nu_{\tau}$ at Belle, Phys. Rev. D 82, 072005 (2010).

[25] M. Huschle et al. (Belle Collaboration), Measurement of the branching ratio of $\bar{B} \rightarrow D^{(*)} \tau^{-} \bar{\nu}_{\tau}$ relative to $\bar{B} \rightarrow D^{(*)} \ell^{-} \bar{\nu}_{\ell}$ decays with hadronic tagging at Belle, Phys. Rev. D 92, 072014 (2015).

[26] S. Hirose et al. (Belle Collaboration), Measurement of the $\tau$ Lepton Polarization and $R\left(D^{*}\right)$ in the Decay $\bar{B} \rightarrow D^{*} \tau^{-} \bar{\nu}_{\tau}$, Phys. Rev. Lett. 118, 211801 (2017).

[27] LHCb Collaboration, Measurement of Form-FactorIndependent Observables in the Decay $B^{0} \rightarrow K^{* 0} \mu^{+} \mu^{-}$, Phys. Rev. Lett. 111, 191801 (2013).

[28] LHCb Collaboration, Test of Lepton Universality Using $B^{+} \rightarrow K^{+} \ell^{+} \ell^{-}$Decays, Phys. Rev. Lett. 113, 151601 (2014).

[29] LHCb Collaboration, Measurement of the Ratio of Branching Fractions $\mathcal{B}\left(\bar{B}^{0} \rightarrow D^{*+} \tau^{-} \bar{\nu}_{\tau}\right) / \mathcal{B}\left(\bar{B}^{0} \rightarrow D^{*+} \mu^{-} \bar{\nu}_{\mu}\right)$, Phys. Rev. Lett. 115, 111803 (2015); Erratum, Phys. Rev. Lett.115, 159901(E) (2015).

[30] LHCb Collaboration, Angular analysis of the $B^{0} \rightarrow K^{* 0} \mu^{+} \mu^{-}$ decay using $3 \mathrm{fb}^{-1}$ of integrated luminosity, J. High Energy Phys. 02 (2016) 104.

[31] LHCb Collaboration, Test of lepton universality with $B^{0} \rightarrow K^{* 0} \ell^{+} \ell^{-}$decays, J. High Energy Phys. 08 (2017) 055.

[32] M. Tanaka and R. Watanabe, New physics in the weak interaction of $\bar{B} \rightarrow D^{(*)} \tau \bar{\nu}$, Phys. Rev. D 87, 034028 (2013).

[33] Y. Sakaki, M. Tanaka, A. Tayduganov, and R. Watanabe, Testing leptoquark models in $\bar{B} \rightarrow D^{(*)} \tau \bar{\nu}$, Phys. Rev. D 88, 094012 (2013).

[34] I. Doršner, S. Fajfer, N. Košnik, and I. Nišandžić, Minimally flavored colored scalar in $\bar{B} \rightarrow D^{(*)} \tau \bar{\nu}$ and the mass matrices constraints, J. High Energy Phys. 11 (2013) 084.

[35] B. Gripaios, M. Nardecchia, and S. A. Renner, Composite leptoquarks and anomalies in $B$-meson decays, J. High Energy Phys. 05 (2015) 006.

[36] M. Bauer and M. Neubert, Minimal Leptoquark Explanation for the $\mathrm{R}_{D^{(*)}}, \mathrm{R}_{K}$, and $(g-2)_{\mu}$ Anomalies, Phys. Rev. Lett. 116, 141802 (2016).

[37] D. Buttazzo, A. Greljo, G. Isidori, and D. Marzocca, B-physics anomalies: a guide to combined explanations, J. High Energy Phys. 11 (2017) 044.

[38] I. Doršner and A. Greljo, Leptoquark toolbox for precision collider studies, J. High Energy Phys. 05 (2018) 126.

[39] B. Diaz, M. Schmaltz, and Y.-M. Zhong, The leptoquark hunter's guide: Pair production, J. High Energy Phys. 10 (2017) 097.

[40] ATLAS Collaboration, Searches for scalar leptoquarks in pp collisions at $\sqrt{s}=8 \mathrm{TeV}$ with the ATLAS detector, Eur. Phys. J. C 76, 5 (2016).

[41] CMS Collaboration, Search for third-generation scalar leptoquarks decaying to a top quark and a $\tau$ lepton at $\sqrt{s}=13 \mathrm{TeV}$, arXiv:1803.02864.
[42] CMS Collaboration, Search for pair production of first and second generation leptoquarks in proton-proton collisions at $\sqrt{s}=8 \mathrm{TeV}$, Phys. Rev. D 93, 032004 (2016).

[43] CMS Collaboration, Search for pair production of thirdgeneration scalar leptoquarks and top squarks in protonproton collisions at $\sqrt{s}=8 \mathrm{TeV}$, Phys. Lett. B 739, 229 (2014).

[44] T. Aaltonen et al. (CDF Collaboration), Search for New Physics with a Dijet Plus Missing Transverse Energy Signature in $p \bar{p}$ Collisions at $\sqrt{s}=1.96 \mathrm{TeV}$, Phys. Rev. Lett. 105, 131801 (2010).

[45] D. Acosta et al. (CDF Collaboration), Search for firstgeneration scalar leptoquarks in $p \bar{p}$ collisions at $\sqrt{s}=$ 1.96 TeV, Phys. Rev. D 72, 051107 (2005).

[46] A. Abulencia et al. (CDF Collaboration), Search for second-generation scalar leptoquarks in $p \bar{p}$ collisions at $\sqrt{s}=1.96 \mathrm{TeV}$, Phys. Rev. D 73, 051102 (2006).

[47] V. M. Abazov et al. (D0 Collaboration), Search for scalar leptoquarks and $T$-odd quarks in the acoplanar jet topology using $2.5 \mathrm{fb}^{-1}$ of $p \bar{p}$ collision data at $\sqrt{s}=1.96 \mathrm{TeV}$, Phys. Lett. B 668, 357 (2008).

[48] V. M. Abazov et al. (D0 Collaboration), Search for first generation leptoquark pair production in the electron + missing energy + jets final state, Phys. Rev. D 84, 071104 (2011).

[49] V. M. Abazov et al. (D0 Collaboration), Search for pair production of second generation scalar leptoquarks, Phys. Lett. B 671, 224 (2009).

[50] F. D. Aaron et al. (H1 Collaboration), Search for first generation leptoquarks in ep collisions at HERA, Phys. Lett. B 704, 388 (2011).

[51] H. Abramowicz et al. (ZEUS Collaboration), Search for first-generation leptoquarks at HERA, Phys. Rev. D 86, 012005 (2012).

[52] ATLAS Collaboration, Search for third generation scalar leptoquarks in pp collisions at $\sqrt{s}=7 \mathrm{TeV}$ with the ATLAS detector, J. High Energy Phys. 06 (2013) 033.

[53] CMS Collaboration, Search for third-generation scalar leptoquarks and heavy right-handed neutrinos in final states with two tau leptons and two jets in proton-proton collisions at $\sqrt{s}=13 \mathrm{TeV}$, J. High Energy Phys. 07 (2017) 121.

[54] T. Aaltonen et al. (CDF), Search for Pair Production of Scalar Top Quarks Decaying to a $\tau$ Lepton and a $b$ Quark in $p \bar{p}$ Collisions at $\sqrt{s}=1.96 \mathrm{TeV}$, Phys. Rev. Lett. 101, 071802 (2008).

[55] T. Aaltonen et al. (CDF Collaboration), Search for third generation vector leptoquarks in $p \bar{p}$ collisions at $\sqrt{s}=1.96$ TeV, Phys. Rev. D 77, 091105 (2008).

[56] V. M. Abazov et al. (D0 Collaboration), Search for Third Generation Scalar Leptoquarks Decaying into $\tau b$, Phys. Rev. Lett. 101, 241802 (2008).

[57] C. G. Lester and D. J. Summers, Measuring masses of semiinvisibly decaying particles pair produced at hadron colliders, Phys. Lett. B 463, 99 (1999).

[58] CMS Collaboration, Search for new phenomena with the $M_{\mathrm{T} 2}$ variable in the all-hadronic final state produced in proton-proton collisions at $\sqrt{s}=13 \mathrm{TeV}$, Eur. Phys. J. C 77, 710 (2017).

[59] CMS Collaboration, The CMS experiment at the CERN LHC, J. Instrum. 3, S08004 (2008). 
[60] CMS Collaboration, Particle-flow reconstruction and global event description with the CMS detector, J. Instrum. 12, P10003 (2017).

[61] M. Cacciari, G. P. Salam, and G. Soyez, The anti- $k_{\mathrm{T}}$ jet clustering algorithm, J. High Energy Phys. 04 (2008) 063.

[62] M. Cacciari, G. P. Salam, and G. Soyez, FastJet user manual, Eur. Phys. J. C 72, 1896 (2012).

[63] CMS Collaboration, Identification of heavy-flavour jets with the CMS detector in pp collisions at $13 \mathrm{TeV}$, J. Instrum. 13, P05011 (2018).

[64] J. Alwall, R. Frederix, S. Frixione, V. Hirschi, F. Maltoni, O. Mattelaer, H.-S. Shao, T. Stelzer, P. Torrielli, and M. Zaro, The automated computation of tree-level and next-to-leading order differential cross sections, and their matching to parton shower simulations, J. High Energy Phys. 07 (2014) 079.

[65] R. D. Ball et al. (NNPDF Collaboration), Parton distributions for the LHC run II, J. High Energy Phys. 04 (2015) 040.

[66] T. Sjöstrand, S. Ask, J. R. Christiansen, R. Corke, N. Desai, P. Ilten, S. Mrenna, S. Prestel, C. O. Rasmussen, and P. Z. Skands, An introduction to PYTHIA 8.2, Comput. Phys. Commun. 191, 159 (2015).

[67] CMS Collaboration, Event generator tunes obtained from underlying event and multiparton scattering measurements, Eur. Phys. J. C 76, 155 (2016).

[68] J. Alwall et al., Comparative study of various algorithms for the merging of parton showers and matrix elements in hadronic collisions, Eur. Phys. J. C 53, 473 (2008).

[69] S. Agostinelli et al. (GEANT4 Collaboration), GEANT4-A simulation toolkit, Nucl. Instrum. Methods Phys. Res., Sect. A 506, 250 (2003).

[70] CMS Collaboration, The fast simulation of the CMS detector at LHC, J. Phys. Conf. Ser. 331, 032049 (2011).

[71] N. Arkani-Hamed, P. Schuster, N. Toro, J. Thaler, L.-T. Wang, B. Knuteson, and S. Mrenna, MARMOSET: The path from LHC data to the new standard model via on-shell effective theories, arXiv:hep-ph/0703088.
[72] J. Alwall, P. C. Schuster, and N. Toro, Simplified models for a first characterization of new physics at the LHC, Phys. Rev. D 79, 075020 (2009).

[73] J. Alwall, M.-P. Le, M. Lisanti, and J. G. Wacker, Modelindependent jets plus missing energy searches, Phys. Rev. D 79, 015005 (2009).

[74] D. Alves, N. Arkani-Hamed, S. Arora, Y. Bai, M. Baumgart, J. Berger, M. Buckley, B. Butler, S. Chang, H.-C. Cheng, C. Cheung, R. S. Chivukula, W. Sang Cho, R. Cotta, M. D'Alfonso et al., Simplified models for LHC new physics searches, J. Phys. G 39, 105005 (2012).

[75] CMS Collaboration, Interpretation of searches for supersymmetry with simplified models, Phys. Rev. D 88, 052017 (2013).

[76] R. D. Ball et al. (NNPDF Collaboration), Parton distributions with LHC data, Nucl. Phys. B867, 244 (2013).

[77] R. D. Ball et al. (NNPDF Collaboration), Parton distributions from high-precision collider data, Eur. Phys. J. C 77, 663 (2017).

[78] T. Junk, Confidence level computation for combining searches with small statistics, Nucl. Instrum. Methods Phys. Res, Sect. A 434, 435 (1999).

[79] A. L. Read, Presentation of search results: the $\mathrm{CL}_{\mathrm{s}}$ technique, J. Phys. G 28, 2693 (2002).

[80] G. Cowan, K. Cranmer, E. Gross, and O. Vitells, Asymptotic formulae for likelihood-based tests of new physics, Eur. Phys. J. C 71, 1554 (2011); Erratum, Eur. Phys. J. C73, 2501 (2013).

[81] ATLAS and CMS Collaborations, Procedure for the LHC Higgs boson search combination in summer 2011, Technical Report No. ATL-PHYS-PUB-2011-011, CMS-NOTE2011-005, http://cds.cern.ch/record/1379837, 2011.

[82] CMS Collaboration, CMS luminosity measurements for the 2016 data taking period, CMS Physics Analysis Summary, Report No. CMS-PAS-LUM-17-001, CERN, https://cds .cern.ch/record/2257069, 2017.

A. M. Sirunyan, ${ }^{1}$ A. Tumasyan, ${ }^{1}$ W. Adam, ${ }^{2}$ F. Ambrogi, ${ }^{2}$ E. Asilar, ${ }^{2}$ T. Bergauer, ${ }^{2}$ J. Brandstetter, ${ }^{2}$ M. Dragicevic, ${ }^{2}$ J. Erö, ${ }^{2}$ A. Escalante Del Valle, ${ }^{2}$ M. Flechl, ${ }^{2}$ R. Frühwirth, ${ }^{2, b}$ V. M. Ghete, ${ }^{2}$ J. Hrubec, ${ }^{2}$ M. Jeitler, ${ }^{2, b}$ N. Krammer, ${ }^{2}$ I. Krätschmer, ${ }^{2}$ D. Liko, ${ }^{2}$ T. Madlener, ${ }^{2}$ I. Mikulec, ${ }^{2}$ N. Rad, ${ }^{2}$ H. Rohringer, ${ }^{2}$ J. Schieck, ${ }^{2, b}$ R. Schöfbeck, ${ }^{2}$ M. Spanring, ${ }^{2}$ D. Spitzbart, ${ }^{2}$ A. Taurok, ${ }^{2}$ W. Waltenberger, ${ }^{2}$ J. Wittmann, ${ }^{2}$ C.-E. Wulz, ${ }^{2, b}$ M. Zarucki, ${ }^{2}$ V. Chekhovsky, ${ }^{3}$ V. Mossolov, ${ }^{3}$ J. Suarez Gonzalez, ${ }^{3}$ E. A. De Wolf, ${ }^{4}$ D. Di Croce, ${ }^{4}$ X. Janssen, ${ }^{4}$ J. Lauwers, ${ }^{4}$ M. Pieters, ${ }^{4}$ M. Van De Klundert, ${ }^{4}$ H. Van Haevermaet, ${ }^{4}$ P. Van Mechelen, ${ }^{4}$ N. Van Remortel, ${ }^{4}$ S. Abu Zeid, ${ }^{5}$ F. Blekman, ${ }^{5}$ J. D'Hondt, ${ }^{5}$ I. De Bruyn, ${ }^{5}$ J. De Clercq, ${ }^{5}$ K. Deroover, ${ }^{5}$ G. Flouris, ${ }^{5}$ D. Lontkovskyi, ${ }^{5}$ S. Lowette, ${ }^{5}$ I. Marchesini ${ }^{5}$ S. Moortgat,${ }^{5}$ L. Moreels, ${ }^{5}$ Q. Python, ${ }^{5}$ K. Skovpen, ${ }^{5}$ S. Tavernier, ${ }^{5}$ W. Van Doninck, ${ }^{5}$ P. Van Mulders, ${ }^{5}$ I. Van Parijs, ${ }^{5}$ D. Beghin,,${ }^{6}$ B. Bilin, ${ }^{6}$ H. Brun, ${ }^{6}$ B. Clerbaux, ${ }^{6}$ G. De Lentdecker, ${ }^{6}$ H. Delannoy, ${ }_{6}^{6}$ B. Dorney, ${ }^{6}$ G. Fasanella, ${ }^{6}$ L. Favart, ${ }^{6}$ R. Goldouzian, ${ }^{6}$ A. Grebenyuk, ${ }^{6}$ A. K. Kalsi, ${ }^{6}$ T. Lenzi, ${ }^{6}$ J. Luetic, ${ }^{6}$ N. Postiau, ${ }^{6}$ E. Starling, ${ }^{6}$ L. Thomas, ${ }^{6}$ C. Vander Velde, ${ }^{6}$ P. Vanlaer, ${ }^{6}$ D. Vannerom, ${ }^{6}$ Q. Wang, ${ }^{6}$ T. Cornelis, ${ }^{7}$ D. Dobur, ${ }^{7}$ A. Fagot, ${ }^{7}$ M. Gul, ${ }^{7}$ I. Khvastunov, ${ }^{7, \mathrm{c}}$ D. Poyraz, ${ }^{7}$ C. Roskas, ${ }^{7}$ D. Trocino, ${ }^{7}$ M. Tytgat, ${ }^{7}$ W. Verbeke, ${ }^{7}$ B. Vermassen, ${ }^{7}$ M. Vit, ${ }^{7}$ N. Zaganidis, ${ }^{7}$ H. Bakhshiansohi, ${ }^{8}$ O. Bondu, ${ }^{8}$ S. Brochet, ${ }^{8}$ G. Bruno, ${ }^{8}$ C. Caputo, ${ }^{8}$ P. David, ${ }^{8}$ C. Delaere, ${ }^{8}$ M. Delcourt, ${ }^{8}$ B. Francois,${ }^{8}$ A. Giammanco, ${ }^{8}$ G. Krintiras, ${ }^{8}$ V. Lemaitre, ${ }^{8}$ A. Magitteri, ${ }^{8}$ A. Mertens, ${ }^{8}$ M. Musich ${ }^{8}$ K. Piotrzkowski, ${ }^{8}$ A. Saggio, ${ }^{8}$ M. Vidal Marono, ${ }^{8}$ S. Wertz, ${ }^{8}$ J. Zobec, ${ }^{8}$ F. L. Alves, ${ }^{9}$ G. A. Alves, ${ }^{9}$ L. Brito, ${ }^{9}$ M. Correa Martins Junior, ${ }^{9}$ G. Correia Silva, ${ }^{9}$ C. Hensel, ${ }^{9}$ A. Moraes, ${ }^{9}$ M. E. Pol, ${ }^{9}$ P. Rebello Teles, ${ }^{9}$

E. Belchior Batista Das Chagas, ${ }^{10}$ W. Carvalho, ${ }^{10}$ J. Chinellato, ${ }^{10, \mathrm{~d}}$ E. Coelho, ${ }^{10}$ E. M. Da Costa, ${ }^{10}$ G. G. Da Silveira,,${ }^{10, e}$ 
D. De Jesus Damiao, ${ }^{10}$ C. De Oliveira Martins, ${ }^{10}$ S. Fonseca De Souza, ${ }^{10}$ H. Malbouisson, ${ }^{10}$ D. Matos Figueiredo, ${ }^{10}$ M. Melo De Almeida, ${ }^{10}$ C. Mora Herrera, ${ }^{10}$ L. Mundim, ${ }^{10}$ H. Nogima, ${ }^{10}$ W. L. Prado Da Silva, ${ }^{10}$ L. J. Sanchez Rosas, ${ }^{10}$ A. Santoro, ${ }^{10}$ A. Sznajder, ${ }^{10}$ M. Thiel,${ }^{10}$ E. J. Tonelli Manganote,${ }^{10, d}$ F. Torres Da Silva De Araujo, ${ }^{10}$ A. Vilela Pereira, ${ }^{10}$ S. Ahuja, ${ }^{11 \mathrm{a}}$ C. A. Bernardes,${ }^{11 \mathrm{a}}$ L. Calligaris, ${ }^{11 \mathrm{a}}$ T. R. Fernandez Perez Tomei, ${ }^{11 \mathrm{a}}$ E. M. Gregores, ${ }^{11 \mathrm{a}, 1 \mathrm{~b}}$

P. G. Mercadante, ${ }^{11 \mathrm{a}, 11 \mathrm{~b}}$ S. F. Novaes, ${ }^{11 \mathrm{a}}$ Sandra S. Padula, ${ }^{11 \mathrm{a}}$ D. Romero Abad, ${ }^{11 \mathrm{a}, 1 \mathrm{bb}}$ A. Aleksandrov, ${ }^{12}$ R. Hadjiiska, ${ }^{12}$ P. Iaydjiev, ${ }^{12}$ A. Marinov, ${ }^{12}$ M. Misheva, ${ }^{12}$ M. Rodozov, ${ }^{12}$ M. Shopova, ${ }^{12}$ G. Sultanov, ${ }^{12}$ A. Dimitrov, ${ }^{13}$ L. Litov, ${ }^{13}$ B. Pavlov, ${ }^{13}$ P. Petkov, ${ }^{13}$ W. Fang, ${ }^{14, \mathrm{f}}$ X. Gao,${ }^{14, \mathrm{f}}$ L. Yuan, ${ }^{14}$ M. Ahmad, ${ }^{15}$ J. G. Bian, ${ }^{15}$ G. M. Chen, ${ }^{15}$ H. S. Chen, ${ }^{15}$ M. Chen, ${ }^{15}$ Y. Chen, ${ }^{15}$ C. H. Jiang, ${ }^{15}$ D. Leggat,${ }^{15}$ H. Liao ${ }^{15}$ Z. Liu, ${ }^{15}$ F. Romeo,${ }^{15}$ S. M. Shaheen, ${ }^{15, g}$ A. Spiezia, ${ }^{15}$ J. Tao,${ }^{15}$ C. Wang, ${ }^{15}$ Z. Wang, ${ }^{15}$ E. Yazgan, ${ }^{15}$ H. Zhang, ${ }^{15}$ J. Zhao, ${ }^{15}$ Y. Ban, ${ }^{16}$ G. Chen, ${ }^{16}$ A. Levin, ${ }^{16}$ J. Li, ${ }^{16}$ L. Li, ${ }^{16}$ Q. Li, ${ }^{16}$ Y. Mao, ${ }^{16}$ S. J. Qian, ${ }^{16}$ D. Wang, ${ }^{16}$ Z. Xu, ${ }^{16}$ Y. Wang, ${ }^{17}$ C. Avila, ${ }^{18}$ A. Cabrera, ${ }^{18}$ C. A. Carrillo Montoya, ${ }^{18}$

L. F. Chaparro Sierra, ${ }^{18}$ C. Florez, ${ }^{18}$ C. F. González Hernández, ${ }^{18}$ M. A. Segura Delgado, ${ }^{18}$ B. Courbon, ${ }^{19}$ N. Godinovic, ${ }^{19}$ D. Lelas, ${ }^{19}$ I. Puljak,${ }^{19}$ T. Sculac, ${ }^{19}$ Z. Antunovic, ${ }^{20}$ M. Kovac, ${ }^{20}$ V. Brigljevic, ${ }^{21}$ D. Ferencek, ${ }^{21}$ K. Kadija, ${ }^{21}$ B. Mesic, ${ }^{21}$ A. Starodumov, ${ }^{21, h}$ T. Susa, ${ }^{21}$ M. W. Ather ${ }^{22}$ A. Attikis, ${ }^{22}$ M. Kolosova, ${ }^{22}$ G. Mavromanolakis, ${ }^{22}$ J. Mousa, ${ }^{22}$ C. Nicolaou, ${ }^{22}$ F. Ptochos, ${ }^{22}$ P. A. Razis, ${ }^{22}$ H. Rykaczewski, ${ }^{22}$ M. Finger, ${ }^{23, i}$ M. Finger Jr., ${ }^{23, i}$ E. Ayala, ${ }^{24}$ E. Carrera Jarrin, ${ }^{25}$ Y. Assran, ${ }^{26, j, k}$ S. Khalil, ${ }^{26,1}$ A. Mahrous, ${ }^{26, m}$ S. Bhowmik, ${ }^{27}$ A. Carvalho Antunes De Oliveira, ${ }^{27}$ R. K. Dewanjee, ${ }^{27}$ K. Ehataht, ${ }^{27}$ M. Kadastik, ${ }^{27}$ M. Raidal, ${ }^{27}$ C. Veelken, ${ }^{27}$ P. Eerola, ${ }^{28}$ H. Kirschenmann, ${ }^{28}$ J. Pekkanen, ${ }^{28}$ M. Voutilainen, ${ }^{28}$

J. Havukainen, ${ }^{29}$ J. K. Heikkilä, ${ }^{29}$ T. Järvinen, ${ }^{29}$ V. Karimäki, ${ }^{29}$ R. Kinnunen, ${ }^{29}$ T. Lampén, ${ }^{29}$ K. Lassila-Perini, ${ }^{29}$ S. Laurila, ${ }^{29}$ S. Lehti, ${ }^{29}$ T. Lindén, ${ }^{29}$ P. Luukka, ${ }^{29}$ T. Mäenpää, ${ }^{29}$ H. Siikonen, ${ }^{29}$ E. Tuominen,,${ }^{29}$ J. Tuominiemi, ${ }^{29}$ T. Tuuva,${ }^{30}$ M. Besancon, ${ }^{31}$ F. Couderc, ${ }^{31}$ M. Dejardin, ${ }^{31}$ D. Denegri, ${ }^{31}$ J. L. Faure, ${ }^{31}$ F. Ferri, ${ }^{31}$ S. Ganjour, ${ }^{31}$ A. Givernaud, ${ }^{31}$ P. Gras,${ }^{31}$ G. Hamel de Monchenault, ${ }^{31}$ P. Jarry, ${ }^{31}$ C. Leloup,${ }^{31}$ E. Locci,${ }^{31}$ J. Malcles,${ }^{31}$ G. Negro, ${ }^{31}$ J. Rander, ${ }^{31}$ A. Rosowsky, ${ }^{31}$ M. Ö. Sahin, ${ }^{31}$ M. Titov, ${ }^{31}$ A. Abdulsalam, ${ }^{32, n}$ C. Amendola, ${ }^{32}$ I. Antropov, ${ }^{32}$ F. Beaudette, ${ }^{32}$ P. Busson, ${ }^{32}$ C. Charlot, ${ }^{32}$ R. Granier de Cassagnac, ${ }^{32}$ I. Kucher, ${ }^{32}$ S. Lisniak, ${ }^{32}$ A. Lobanov, ${ }^{32}$ J. Martin Blanco, ${ }^{32}$ M. Nguyen, ${ }^{32}$ C. Ochando, ${ }^{32}$ G. Ortona, ${ }^{32}$ P. Pigard, ${ }^{32}$ R. Salerno, ${ }^{32}$ J. B. Sauvan, ${ }^{32}$ Y. Sirois, ${ }^{32}$ A. G. Stahl Leiton, ${ }^{32}$ A. Zabi, ${ }^{32}$ A. Zghiche, ${ }^{32}$ J.-L. Agram, ${ }^{33,0}$ J. Andrea, ${ }^{33}$ D. Bloch, ${ }^{33}$ J.-M. Brom, ${ }^{33}$ E. C. Chabert, ${ }^{33}$ V. Cherepanov, ${ }^{33}$ C. Collard,${ }^{33}$ E. Conte, ${ }^{33, o}$ J.-C. Fontaine, ${ }^{33,0}$ D. Gelé, ${ }^{33}$ U. Goerlach ${ }^{33}$ M. Jansová, ${ }^{33}$ A.-C. Le Bihan, ${ }^{33}$ N. Tonon, ${ }^{33}$ P. Van Hove, ${ }^{33}$ S. Gadrat,${ }^{34}$ S. Beauceron, ${ }^{35}$ C. Bernet, ${ }^{35}$ G. Boudoul,${ }^{35}$ N. Chanon, ${ }^{35}$ R. Chierici, ${ }^{35}$ D. Contardo, ${ }^{35}$ P. Depasse, ${ }^{35}$ H. El Mamouni, ${ }^{35}$ J. Fay, ${ }^{35}$ L. Finco, ${ }^{35}$ S. Gascon, ${ }^{35}$ M. Gouzevitch, ${ }^{35}$ G. Grenier, ${ }^{35}$ B. Ille, ${ }^{35}$ F. Lagarde, ${ }^{35}$ I. B. Laktineh,${ }^{35}$ H. Lattaud, ${ }^{35}$ M. Lethuillier, ${ }^{35}$ L. Mirabito, ${ }^{35}$ A. L. Pequegnot,${ }^{35}$ S. Perries, ${ }^{35}$ A. Popov, ${ }^{35, p}$ V. Sordini, ${ }^{35}$ M. Vander Donckt, ${ }^{35}$ S. Viret,${ }^{35}$ S. Zhang, ${ }^{35}$ A. Khvedelidze, ${ }^{36, i}$ Z. Tsamalaidze, ${ }^{37, i}$ C. Autermann, ${ }^{38}$ L. Feld ${ }^{38}$ M. K. Kiesel, ${ }^{38}$ K. Klein, ${ }^{38}$ M. Lipinski, ${ }^{38}$ M. Preuten,${ }^{38}$ M. P. Rauch, ${ }^{38}$ C. Schomakers, ${ }^{38}$ J. Schulz,${ }^{38}$ M. Teroerde, ${ }^{38}$ B. Wittmer, ${ }^{38}$ V. Zhukov, ${ }^{38, p}$ A. Albert, ${ }^{39}$ D. Duchardt, ${ }^{39}$ M. Endres,${ }^{39}$ M. Erdmann,${ }^{39}$ T. Esch, ${ }^{39}$ R. Fischer, ${ }^{39}$ S. Ghosh, ${ }^{39}$ A. Güth, ${ }^{39}$ T. Hebbeker, ${ }^{39}$ C. Heidemann, ${ }^{39}$ K. Hoepfner, ${ }^{39}$ H. Keller, ${ }^{39}$ S. Knutzen, ${ }^{39}$ L. Mastrolorenzo, ${ }^{39}$ M. Merschmeyer, ${ }^{39}$ A. Meyer, ${ }^{39}$ P. Millet, ${ }^{39}$ S. Mukherjee, ${ }^{39}$

T. Pook, ${ }^{39}$ M. Radziej,${ }^{39}$ H. Reithler, ${ }^{39}$ M. Rieger, ${ }^{39}$ F. Scheuch, ${ }^{39}$ A. Schmidt, ${ }^{39}$ D. Teyssier, ${ }^{39}$ G. Flügge, ${ }^{40}$

O. Hlushchenko, ${ }^{40}$ B. Kargoll, ${ }^{40}$ T. Kress, ${ }^{40}$ A. Künsken, ${ }^{40}$ T. Müller, ${ }^{40}$ A. Nehrkorn, ${ }^{40}$ A. Nowack, ${ }^{40}$ C. Pistone, ${ }^{40}$ O. Pooth, ${ }^{40}$ H. Sert, ${ }^{40}$ A. Stahl,${ }^{40, q}$ M. Aldaya Martin, ${ }^{41}$ T. Arndt,${ }^{41}$ C. Asawatangtrakuldee, ${ }^{41}$ I. Babounikau, ${ }^{41}$

K. Beernaert, ${ }^{41}$ O. Behnke, ${ }^{41}$ U. Behrens, ${ }^{41}$ A. Bermúdez Martínez,${ }^{41}$ D. Bertsche, ${ }^{41}$ A. A. Bin Anuar, ${ }^{41}$ K. Borras, ${ }^{41, r}$ V. Botta, ${ }^{41}$ A. Campbell, ${ }^{41}$ P. Connor, ${ }^{41}$ C. Contreras-Campana, ${ }^{41}$ F. Costanza, ${ }^{41}$ V. Danilov, ${ }^{41}$ A. De Wit, ${ }^{41}$ M. M. Defranchis, ${ }^{41}$ C. Diez Pardos, ${ }^{41}$ D. Domínguez Damiani, ${ }^{41}$ G. Eckerlin, ${ }^{41}$ T. Eichhorn, ${ }^{41}$ A. Elwood ${ }^{41}$ E. Eren, ${ }^{41}$ E. Gallo, ${ }^{41, s}$ A. Geiser, ${ }^{41}$ J. M. Grados Luyando, ${ }^{41}$ A. Grohsjean, ${ }^{41}$ P. Gunnellini, ${ }^{41}$ M. Guthoff, ${ }^{41}$ M. Haranko, ${ }^{41}$ A. Harb,${ }^{41}$ J. Hauk, ${ }^{41}$ H. Jung, ${ }^{41}$ M. Kasemann, ${ }^{41}$ J. Keaveney, ${ }^{41}$ C. Kleinwort, ${ }^{41}$ J. Knolle, ${ }^{41}$ D. Krücker, ${ }^{41}$ W. Lange, ${ }^{41}$ A. Lelek,${ }^{41}$ T. Lenz,${ }^{41}$ K. Lipka, ${ }^{41}$ W. Lohmann,${ }^{41, t}$ R. Mankel ${ }^{41}$ I.-A. Melzer-Pellmann, ${ }^{41}$ A. B. Meyer, ${ }^{41}$ M. Meyer ${ }^{41}$ M. Missiroli, ${ }^{41}$ G. Mittag, ${ }^{41}$ J. Mnich, ${ }^{41}$ V. Myronenko, ${ }^{41}$ S. K. Pflitsch, ${ }^{41}$ D. Pitzl,${ }^{41}$ A. Raspereza, ${ }^{41}$ M. Savitskyi, ${ }^{41}$ P. Saxena, ${ }^{41}$ P. Schütze, ${ }^{41}$ C. Schwanenberger, ${ }^{41}$ R. Shevchenko, ${ }^{41}$ A. Singh, ${ }^{41}$ N. Stefaniuk,${ }^{41}$ H. Tholen, ${ }^{41}$ O. Turkot,${ }^{41}$ A. Vagnerini, ${ }^{41}$ G. P. Van Onsem, ${ }^{41}$ R. Walsh, ${ }^{41}$ Y. Wen, ${ }^{41}$ K. Wichmann, ${ }^{41}$ C. Wissing, ${ }^{41}$ O. Zenaiev, ${ }^{41}$ R. Aggleton, ${ }^{42}$ S. Bein, ${ }^{42}$ L. Benato, ${ }^{42}$ A. Benecke, ${ }^{42}$ V. Blobel, ${ }^{42}$ M. Centis Vignali, ${ }^{42}$ T. Dreyer, ${ }^{42}$ E. Garutti, ${ }^{42}$ D. Gonzalez ${ }^{42}$ J. Haller, ${ }^{42}$ A. Hinzmann, ${ }^{42}$ A. Karavdina, ${ }^{42}$ G. Kasieczka, ${ }^{42}$ R. Klanner, ${ }^{42}$ R. Kogler, ${ }^{42}$ N. Kovalchuk, ${ }^{42}$ S. Kurz, ${ }^{42}$ V. Kutzner, ${ }^{42}$ J. Lange, ${ }^{42}$ D. Marconi, ${ }^{42}$ J. Multhaup, ${ }^{42}$ M. Niedziela, ${ }^{42}$ D. Nowatschin ${ }^{42}$ A. Perieanu, ${ }^{42}$ A. Reimers, ${ }^{42}$ O. Rieger, ${ }^{42}$ C. Scharf, ${ }^{42}$ P. Schleper, ${ }^{42}$ S. Schumann, ${ }^{42}$ J. Schwandt ${ }^{42}$ J. Sonneveld, ${ }^{42}$ H. Stadie, ${ }^{42}$ G. Steinbrück, ${ }^{42}$ F. M. Stober, ${ }^{42}$ M. Stöver, ${ }^{42}$ D. Troendle, ${ }^{42}$ A. Vanhoefer, ${ }^{42}$ B. Vormwald, ${ }^{42}$ M. Akbiyik, ${ }^{43}$ C. Barth, ${ }^{43}$ M. Baselga, ${ }^{43}$ S. Baur, ${ }^{43}$ E. Butz, ${ }^{43}$ 
R. Caspart ${ }^{43}$ T. Chwalek, ${ }^{43}$ F. Colombo, ${ }^{43}$ W. De Boer, ${ }^{43}$ A. Dierlamm, ${ }^{43}$ K. El Morabit, ${ }^{43}$ N. Faltermann,${ }^{43}$ B. Freund, ${ }^{43}$ M. Giffels, ${ }^{43}$ M. A. Harrendorf, ${ }^{43}$ F. Hartmann, ${ }^{43, q}$ S. M. Heindl, ${ }^{43}$ U. Husemann, ${ }^{43}$ F. Kassel,${ }^{43, q}$ I. Katkov, ${ }^{43, p}$ S. Kudella, ${ }^{43}$ H. Mildner, ${ }^{43}$ S. Mitra, ${ }^{43}$ M. U. Mozer, ${ }^{43}$ Th. Müller, ${ }^{43}$ M. Plagge, ${ }^{43}$ G. Quast, ${ }^{43}$ K. Rabbertz, ${ }^{43}$ M. Schröder, ${ }^{43}$ I. Shvetsov, ${ }^{43}$ G. Sieber, ${ }^{43}$ H. J. Simonis, ${ }^{43}$ R. Ulrich,${ }^{43}$ S. Wayand, ${ }^{43}$ M. Weber ${ }^{43}$ T. Weiler ${ }^{43}$ S. Williamson, ${ }^{43}$ C. Wöhrmann, ${ }^{43}$ R. Wolf, ${ }^{43}$ G. Anagnostou, ${ }^{44}$ G. Daskalakis, ${ }^{44}$ T. Geralis, ${ }^{44}$ A. Kyriakis,${ }^{44}$ D. Loukas, ${ }^{44}$ G. Paspalaki, ${ }^{44}$ I. Topsis-Giotis, ${ }^{44}$

G. Karathanasis, ${ }^{45}$ S. Kesisoglou, ${ }^{45}$ P. Kontaxakis, ${ }^{45}$ A. Panagiotou, ${ }^{45}$ N. Saoulidou, ${ }^{45}$ E. Tziaferi, ${ }^{45}$ K. Vellidis, ${ }^{45}$

K. Kousouris, ${ }^{46}$ I. Papakrivopoulos, ${ }^{46}$ G. Tsipolitis, ${ }^{46}$ I. Evangelou, ${ }^{47}$ C. Foudas,${ }^{47}$ P. Gianneios, ${ }^{47}$ P. Katsoulis, ${ }^{47}$ P. Kokkas, ${ }^{47}$ S. Mallios ${ }^{47}$ N. Manthos, ${ }^{47}$ I. Papadopoulos, ${ }^{47}$ E. Paradas, ${ }^{47}$ J. Strologas, ${ }^{47}$ F. A. Triantis, ${ }^{47}$ D. Tsitsonis,${ }^{47}$ M. Bartók, ${ }^{48, u}$ M. Csanad, ${ }^{48}$ N. Filipovic, ${ }^{48}$ P. Major, ${ }^{48}$ M. I. Nagy, ${ }^{48}$ G. Pasztor,${ }^{48}$ O. Surányi, ${ }^{48}$ G. I. Veres,${ }^{48}$ G. Bencze,${ }^{49}$ C. Hajdu, ${ }^{49}$ D. Horvath, ${ }^{49, v}$ Á. Hunyadi, ${ }^{49}$ F. Sikler, ${ }^{49}$ T. Á. Vámi, ${ }^{49}$ V. Veszpremi, ${ }^{49}$ G. Vesztergombi, ${ }^{49, a, u}$ N. Beni, ${ }^{50}$

S. Czellar, ${ }^{50}$ J. Karancsi, ${ }^{50, w}$ A. Makovec, ${ }^{50}$ J. Molnar, ${ }^{50}$ Z. Szillasi, ${ }^{50}$ P. Raics,${ }^{51}$ Z. L. Trocsanyi, ${ }^{51}$ B. Ujvari, ${ }^{51}$

S. Choudhury, ${ }^{52}$ J. R. Komaragiri, ${ }^{52}$ P. C. Tiwari, ${ }^{52}$ S. Bahinipati, ${ }^{53, x}$ C. Kar, $^{53}$ P. Mal, ${ }^{53}$ K. Mandal, ${ }^{53}$ A. Nayak, ${ }^{53, y}$ D. K. Sahoo, ${ }^{53, x}$ S. K. Swain, ${ }^{53}$ S. Bansal,${ }^{54}$ S. B. Beri,${ }^{54}$ V. Bhatnagar, ${ }^{54}$ S. Chauhan,${ }^{54}$ R. Chawla, ${ }^{54}$ N. Dhingra, ${ }^{54}$ R. Gupta, ${ }^{54}$ A. Kaur, ${ }^{54}$ A. Kaur, ${ }^{54}$ M. Kaur,${ }^{54}$ S. Kaur, ${ }^{54}$ R. Kumar, ${ }^{54}$ P. Kumari, ${ }^{54}$ M. Lohan, ${ }^{54}$ A. Mehta, ${ }^{54}$ K. Sandeep,${ }^{54}$

S. Sharma, ${ }^{54}$ J. B. Singh,${ }^{54}$ G. Walia,${ }^{54}$ A. Bhardwaj, ${ }^{55}$ B. C. Choudhary, ${ }^{55}$ R. B. Garg, ${ }^{55}$ M. Gola, ${ }^{55}$ S. Keshri, ${ }^{55}$ Ashok Kumar, ${ }^{55}$ S. Malhotra, ${ }^{55}$ M. Naimuddin, ${ }^{55}$ P. Priyanka, ${ }^{55}$ K. Ranjan, ${ }^{55}$ Aashaq Shah, ${ }^{55}$ R. Sharma,${ }^{55}$ R. Bhardwaj, ${ }^{56, z}$ M. Bharti, ${ }^{56}$ R. Bhattacharya ${ }^{56}$ S. Bhattacharya ${ }^{56}$ U. Bhawandeep, ${ }^{56, z}$ D. Bhowmik, ${ }^{56}$ S. Dey, ${ }^{56}$ S. Dutt,${ }^{56, z}$ S. Dutta, ${ }^{56}$ S. Ghosh, ${ }^{56}$ K. Mondal, ${ }^{56}$ S. Nandan, ${ }^{56}$ A. Purohit, ${ }^{56}$ P. K. Rout, ${ }^{56}$ A. Roy, ${ }^{56}$ S. Roy Chowdhury,${ }^{56}$ S. Sarkar,${ }^{56}$ M. Sharan, ${ }^{56}$ B. Singh, ${ }^{56}$ S. Thakur, ${ }^{56, z}$ P. K. Behera, ${ }^{57}$ R. Chudasama,${ }^{58}$ D. Dutta,${ }^{58}$ V. Jha,${ }^{58}$ V. Kumar, ${ }^{58}$ P. K. Netrakanti, ${ }^{58}$ L. M. Pant,${ }^{58}$ P. Shukla, ${ }^{58}$ T. Aziz, ${ }^{59}$ M. A. Bhat,${ }^{59}$ S. Dugad, ${ }^{59}$ G. B. Mohanty, ${ }^{59}$ N. Sur,${ }^{59}$ B. Sutar, ${ }^{59}$ K. V. Ravindra, ${ }^{59}$ S. Banerjee, ${ }^{60}$

S. Bhattacharya ${ }^{60}$ S. Chatterjee, ${ }^{60}$ P. Das, ${ }^{60}$ M. Guchait, ${ }^{60}$ Sa. Jain, ${ }^{60}$ S. Karmakar, ${ }^{60}$ S. Kumar,${ }^{60}$ M. Maity, ${ }^{60, a a}$

G. Majumder, ${ }^{60}$ K. Mazumdar, ${ }^{60}$ N. Sahoo, ${ }^{60}$ T. Sarkar, ${ }^{60, a a}$ S. Chauhan, ${ }^{61}$ S. Dube, ${ }^{61}$ V. Hegde, ${ }^{61}$ A. Kapoor, ${ }^{61}$

K. Kothekar, ${ }^{61}$ S. Pandey, ${ }^{61}$ A. Rane,${ }^{61}$ S. Sharma, ${ }^{61}$ S. Chenarani, ${ }^{62, b b}$ E. Eskandari Tadavani, ${ }^{62}$ S. M. Etesami, ${ }^{62, b b}$ M. Khakzad, ${ }^{62}$ M. Mohammadi Najafabadi, ${ }^{62}$ M. Naseri, ${ }^{62}$ F. Rezaei Hosseinabadi, ${ }^{62}$ B. Safarzadeh, ${ }^{62, c c}$ M. Zeinali, ${ }^{62}$ M. Felcini, ${ }^{63}$ M. Grunewald, ${ }^{63}$ M. Abbrescia, ${ }^{64 a, 64 b}$ C. Calabria, ${ }^{64 a, 64 b}$ A. Colaleo, ${ }^{64 a}$ D. Creanza, ${ }^{64 a, 64 c}$ L. Cristella, ${ }^{64 a, 64 b}$ N. De Filippis, ${ }^{64,64 \mathrm{c}}$ M. De Palma, ${ }^{64 a, 64 b}$ A. Di Florio,${ }^{64 a, 64 b}$ F. Errico, ${ }^{64 a, 64 b}$ L. Fiore, ${ }^{64 a}$ A. Gelmi, ${ }^{64 a, 64 b}$ G. Iaselli, ${ }^{64 a, 64 c}$ M. Ince, ${ }^{64 a, 64 b}$ S. Lezki, ${ }^{64 a, 64 b}$ G. Maggi, ${ }^{64 a, 64 c}$ M. Maggi,${ }^{64 a}$ G. Miniello, ${ }^{64 a, 64 b}$ S. My, ${ }^{64 a, 64 b}$ S. Nuzzo, ${ }^{64 a, 64 b}$ A. Pompili, ${ }^{64 a, 64 b}$

G. Pugliese, ${ }^{64 a, 64 c}$ R. Radogna,${ }^{64 a}$ A. Ranieri, ${ }^{64 a}$ G. Selvaggi, ${ }^{64 a, 64 b}$ A. Sharma, ${ }^{64 a}$ L. Silvestris, ${ }^{64 a}$ R. Venditti, ${ }^{64 a}$ P. Verwilligen, ${ }^{64 a}$ G. Zito, ${ }^{64 a}$ G. Abbiendi, ${ }^{65 a}$ C. Battilana, ${ }^{65 a, 65 b}$ D. Bonacorsi, ${ }^{65 a, 65 b}$ L. Borgonovi, ${ }^{65 a, 65 b}$

S. Braibant-Giacomelli, ${ }^{65 a, 65 b}$ R. Campanini, ${ }^{65 a, 65 b}$ P. Capiluppi, ${ }^{65 a, 65 b}$ A. Castro, ${ }^{65 a, 65 b}$ F. R. Cavallo, ${ }^{65 a}$ S. S. Chhibra, ${ }^{65 a, 65 b}$ C. Ciocca, ${ }^{65 a}$ G. Codispoti, ${ }^{65 a, 65 b}$ M. Cuffiani, ${ }^{65 a, 65 b}$ G. M. Dallavalle, ${ }^{65 a}$ F. Fabbri, ${ }^{65 a}$ A. Fanfani, ${ }^{65 a, 65 b}$ P. Giacomelli, ${ }^{65 a}$ C. Grandi, ${ }^{65 a}$ L. Guiducci, ${ }^{65 a, 65 b}$ F. Iemmi, ${ }^{65 a, 65 b}$ S. Marcellini, ${ }^{65 a}$ G. Masetti, ${ }^{65 a}$ A. Montanari, ${ }^{65 a}$ F. L. Navarria, ${ }^{65 a, 65 b}$ A. Perrotta, ${ }^{65 a}$ F. Primavera, ${ }^{65 a, 65 b, q}$ A. M. Rossi, ${ }^{65,65 b}$ T. Rovelli, ${ }^{65 a, 65 b}$ G. P. Siroli, ${ }^{65 a, 65 b}$ N. Tosi, ${ }^{65 a}$ S. Albergo, ${ }^{66 a, 66 b}$ A. Di Mattia, ${ }^{66 \mathrm{a}}$ R. Potenza, ${ }^{66 a, 66 \mathrm{~b}}$ A. Tricomi, ${ }^{66 a, 66 \mathrm{~b}}$ C. Tuve, ${ }^{66,66 \mathrm{~b}}$ G. Barbagli, ${ }^{67 \mathrm{a}}$ K. Chatterjee, ${ }^{67 a, 67 b}$ V. Ciulli, ${ }^{67 a, 67 b}$ C. Civinini, ${ }^{67 \mathrm{a}}$ R. D’Alessandro, ${ }^{67,67 \mathrm{~b}}$ E. Focardi, ${ }^{67 \mathrm{a}, 67 \mathrm{~b}}$ G. Latino, ${ }^{67 \mathrm{a}}$ P. Lenzi, ${ }^{67 a, 67 \mathrm{~b}}$ M. Meschini, ${ }^{67 \mathrm{a}}$ S. Paoletti, ${ }^{67 \mathrm{a}}$ L. Russo, ${ }^{67 a, d d}$ G. Sguazzoni, ${ }^{67 a}$ D. Strom, ${ }^{67 a}$ L. Viliani,${ }^{67 a}$ L. Benussi ${ }^{68}$ S. Bianco, ${ }^{68}$ F. Fabbri, ${ }^{68}$ D. Piccolo,${ }^{68}$ F. Ferro, ${ }^{69 a}$ F. Ravera, ${ }^{69 \mathrm{a}, 69 \mathrm{~b}}$ E. Robutti, ${ }^{69 \mathrm{a}}$ S. Tosi, ${ }^{69 \mathrm{a}, 69 \mathrm{~b}}$ A. Benaglia, ${ }^{70 \mathrm{a}}$ A. Beschi, ${ }^{70 a, 70 \mathrm{~b}}$ L. Brianza,${ }^{70 a, 70 \mathrm{~b}}$ F. Brivio, ${ }^{70 \mathrm{a}, 70 \mathrm{~b}}$ V. Ciriolo, ${ }^{70 a, 70 b, q}$ S. Di Guida, ${ }^{70 a, 70 b, q}$ M. E. Dinardo ${ }^{70 a, 70 b}$ S. Fiorendi, ${ }^{70 a, 70 b}$ S. Gennai, ${ }^{70 a}$ A. Ghezzi, ${ }^{70 a, 70 b}$ P. Govoni, ${ }^{70 a, 70 b}$ M. Malberti, ${ }^{70 a, 70 b}$ S. Malvezzi, ${ }^{70 a}$ A. Massironi, ${ }^{70 a, 70 b}$ D. Menasce, ${ }^{70 a}$ L. Moroni, ${ }^{70 a}$ M. Paganoni, ${ }^{70 a, 70 b}$ D. Pedrini, ${ }^{70 a}$ S. Ragazzi ${ }^{70 a, 70 b}$ T. Tabarelli de Fatis, ${ }^{70 a, 70 b}$ D. Zuolo, ${ }^{70 a}$ S. Buontempo, ${ }^{71 a}$ N. Cavallo, ${ }^{71 a, 71 c}$ A. Di Crescenzo, ${ }^{71 a, 71 b}$ F. Fabozzi, ${ }^{71 a, 71 \mathrm{c}}$ F. Fienga, ${ }^{71 \mathrm{a}}$ G. Galati, ${ }^{71 \mathrm{a}}$ A. O. M. Iorio, ${ }^{71 \mathrm{a}, 71 \mathrm{~b}}$ W. A. Khan, ${ }^{71 \mathrm{a}}$ L. Lista, ${ }^{71 \mathrm{a}}$ S. Meola, ${ }^{71 \mathrm{a}, 71 \mathrm{~d}, \mathrm{q}}$ P. Paolucci, ${ }^{71 a, q}$ C. Sciacca, ${ }^{71 a, 71 b}$ E. Voevodina ${ }^{71 a, 71 b}$ P. Azzi,${ }^{72 a}$ N. Bacchetta, ${ }^{72 a}$ D. Bisello, ${ }^{72 a, 72 b}$ A. Boletti, ${ }^{72 a, 72 b}$ A. Bragagnolo, ${ }^{72 a}$ R. Carlin, ${ }^{72 a, 72 b}$ P. Checchia, ${ }^{72 a}$ M. Dall'Osso, ${ }^{72 a, 72 b}$ P. De Castro Manzano, ${ }^{72 a}$ T. Dorigo, ${ }^{72 a}$ U. Dosselli, ${ }^{72 a}$

F. Gasparini, ${ }^{72 a, 72 b}$ U. Gasparini, ${ }^{72 a, 72 b}$ A. Gozzelino, ${ }^{72 a}$ S. Lacaprara, ${ }^{72 a}$ P. Lujan, ${ }^{72 a}$ M. Margoni, ${ }^{72 a, 72 b}$

A. T. Meneguzzo, ${ }^{72 a, 72 b}$ J. Pazzini, ${ }^{72 a, 72 b}$ P. Ronchese, ${ }^{72 a, 72 b}$ R. Rossin, ${ }^{72 a, 72 b}$ F. Simonetto, ${ }^{72 a, 72 b}$ A. Tiko, ${ }^{72 a}$ E. Torassa,${ }^{72 a}$ M. Zanetti, ${ }^{72 a, 72 b}$ P. Zotto, ${ }^{72 a, 72 b}$ G. Zumerle, ${ }^{72 a, 72 b}$ A. Braghieri, ${ }^{73 a}$ A. Magnani, ${ }^{73 a}$ P. Montagna, ${ }^{73 a, 73 b}$ S. P. Ratti,${ }^{73 a, 73 b}$ V. Re, ${ }^{73 a}$ M. Ressegotti, ${ }^{73 a, 73 b}$ C. Riccardi, ${ }^{73 a, 73 b}$ P. Salvini, ${ }^{73 a}$ I. Vai, ${ }^{73 a, 73 b}$ P. Vitulo, ${ }^{73 a, 73 b}$ L. Alunni Solestizi, ${ }^{74 a, 74 b}$

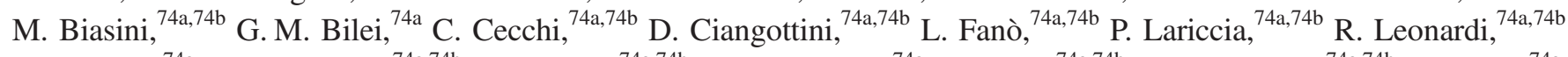
E. Manoni, ${ }^{74 a}$ G. Mantovani, ${ }^{74,74 b}$ V. Mariani, ${ }^{74 a, 74 b}$ M. Menichelli, ${ }^{74 a}$ A. Rossi, ${ }^{74 a, 74 b}$ A. Santocchia, ${ }^{74 a, 74 b}$ D. Spiga, ${ }^{74 a}$ 
K. Androsov, ${ }^{75 a}$ P. Azzurri, ${ }^{75 a}$ G. Bagliesi, ${ }^{75 a}$ L. Bianchini, ${ }^{75 a}$ T. Boccali, ${ }^{75 a}$ L. Borrello, ${ }^{75 a}$ R. Castaldi, ${ }^{75 a}$ M. A. Ciocci, ${ }^{75 a, 75 b}$ R. Dell'Orso, ${ }^{75 a}$ G. Fedi, ${ }^{75 a}$ F. Fiori, ${ }^{75 a, 75 c}$ L. Giannini, ${ }^{75 a, 75 c}$ A. Giassi, ${ }^{75 a}$ M. T. Grippo, ${ }^{75 a}$ F. Ligabue, ${ }^{75 a, 75 c}$ E. Manca, ${ }^{75 a, 75 c}$ G. Mandorli, ${ }^{75 a, 75 c}$ A. Messineo, ${ }^{75 a, 75 b}$ F. Palla, ${ }^{75 a}$ A. Rizzi, ${ }^{75 a, 75 b}$ P. Spagnolo, ${ }^{75 a}$ R. Tenchini, ${ }^{75 a}$ G. Tonelli, ${ }^{75 a, 75 b}$

A. Venturi, ${ }^{75 a}$ P. G. Verdini, ${ }^{75 a}$ L. Barone, ${ }^{76 a, 76 b}$ F. Cavallari, ${ }^{76 a}$ M. Cipriani, ${ }^{76 a, 76 b}$ N. Daci, ${ }^{76 a}$ D. Del Re, ${ }^{76 a, 76 b}$ E. Di Marco, ${ }^{76 a, 76 b}$ M. Diemoz, ${ }^{76 a}$ S. Gelli, ${ }^{76 a, 76 b}$ E. Longo, ${ }^{76 a, 76 b}$ B. Marzocchi, ${ }^{76 a, 76 b}$ P. Meridiani, ${ }^{76 a}$ G. Organtini, ${ }^{76 a, 76 b}$ F. Pandolfi, ${ }^{76 a}$ R. Paramatti, ${ }^{76 a, 76 b}$ F. Preiato, ${ }^{76 a, 76 b}$ S. Rahatlou, ${ }^{76 a, 76 b}$ C. Rovelli, ${ }^{76 a}$ F. Santanastasio, ${ }^{76 a, 76 b}$ N. Amapane, ${ }^{77 a, 77 b}$ R. Arcidiacono, ${ }^{77 a, 77 c}$ S. Argiro, ${ }^{77 a, 77 b}$ M. Arneodo, ${ }^{77 a, 77 c}$ N. Bartosik, ${ }^{77 a}$ R. Bellan, ${ }^{77 a, 77 b}$ C. Biino, ${ }^{77 a}$ N. Cartiglia, ${ }^{77 a}$ F. Cenna, ${ }^{77 a, 77 b}$ S. Cometti, ${ }^{77 a}$ M. Costa, ${ }^{77 a, 77 b}$ R. Covarelli, ${ }^{77 a, 77 b}$ N. Demaria, ${ }^{77 a}$ B. Kiani, ${ }^{77 a, 77 b}$ C. Mariotti, ${ }^{77 a}$ S. Maselli, ${ }^{77 a}$ E. Migliore, ${ }^{77 a, 77 b}$ V. Monaco, ${ }^{77 a, 77 b}$ E. Monteil, ${ }^{77 a, 77 b}$ M. Monteno, ${ }^{77 a}$ M. M. Obertino, ${ }^{77 a, 77 b}$ L. Pacher, ${ }^{77 a, 77 b}$ N. Pastrone, ${ }^{77 a}$ M. Pelliccioni, ${ }^{77 a}$ G. L. Pinna Angioni, ${ }^{77 a, 77 b}$ A. Romero, ${ }^{77 a, 77 b}$ M. Ruspa, ${ }^{77 a, 77 c}$ R. Sacchi, ${ }^{77 a, 77 b}$ K. Shchelina, ${ }^{77 a, 77 b}$ V. Sola, ${ }^{77 a}$ A. Solano, ${ }^{77 a, 77 b}$ D. Soldi, ${ }^{77 a}$ A. Staiano, ${ }^{77 a}$ S. Belforte, ${ }^{78 a}$ V. Candelise, ${ }^{78 a, 78 b}$ M. Casarsa, ${ }^{78 a}$ F. Cossutti, ${ }^{78 a}$ G. Della Ricca, ${ }^{78 a, 78 b}$ F. Vazzoler, ${ }^{78 a, 78 b}$ A. Zanetti, ${ }^{78 a}$ D. H. Kim, ${ }^{79}$ G. N. Kim, ${ }^{79}$ M. S. Kim, ${ }^{79}$ J. Lee, ${ }^{79}$ S. Lee, ${ }^{79}$ S. W. Lee, ${ }^{79}$ C. S. Moon, ${ }^{79}$ Y. D. Oh, ${ }^{79}$ S. Sekmen, ${ }^{79}$ D. C. Son, ${ }^{79}$ Y. C. Yang, ${ }^{79}$ H. Kim, ${ }^{80}$ D. H. Moon, ${ }^{80}$ G. Oh, ${ }^{80}$ J. Goh, ${ }^{81, e e}$ T. J. Kim, ${ }^{81}$ S. Cho, ${ }^{82}$ S. Choi, ${ }^{82}$ Y. Go, ${ }^{82}$ D. Gyun, ${ }^{82}$ S. Ha, ${ }^{82}$ B. Hong, ${ }^{82}$ Y. Jo, ${ }^{82}$ K. Lee, ${ }^{82}$ K. S. Lee, ${ }^{82}$ S. Lee, ${ }^{82}$ J. Lim, ${ }^{82}$ S. K. Park, ${ }^{82}$ Y. Roh, ${ }^{82}$ H. S. Kim, ${ }^{83}$ J. Almond, ${ }^{84}$ J. Kim, ${ }^{84}$ J. S. Kim, ${ }^{84}$ H. Lee, ${ }^{84}$ K. Lee, ${ }^{84}$ K. Nam, ${ }^{84}$ S. B. Oh, ${ }^{84}$ B. C. Radburn-Smith, ${ }^{84}$ S. h. Seo, ${ }^{84}$ U. K. Yang, ${ }^{84}$ H. D. Yoo, ${ }^{84}$ G. B. Yu, ${ }^{84}$ D. Jeon, ${ }^{85}$ H. Kim, ${ }^{85}$ J. H. Kim, ${ }^{85}$ J. S. H. Lee, ${ }^{85}$ I. C. Park, ${ }^{85}$ Y. Choi, ${ }^{86}$ C. Hwang, ${ }^{86}$ J. Lee, ${ }^{86}$ I. Yu, ${ }^{86}$ V. Dudenas, ${ }^{87}$ A. Juodagalvis, ${ }^{87}$ J. Vaitkus, ${ }^{87}$ I. Ahmed, ${ }^{88}$ Z. A. Ibrahim, ${ }^{88}$ M. A. B. Md Ali, ${ }^{88, f f}$ F. Mohamad Idris, ${ }^{88, g g}$ W. A. T. Wan Abdullah, ${ }^{88}$ M. N. Yusli, ${ }^{88}$ Z. Zolkapli, ${ }^{88}$ A. Castaneda Hernandez, ${ }^{89}$ J. A. Murillo Quijada, ${ }^{89}$ M. C. Duran-Osuna, ${ }^{90}$ H. Castilla-Valdez, ${ }^{90}$ E. De La Cruz-Burelo, ${ }^{90}$ G. Ramirez-Sanchez, ${ }^{90}$ I. Heredia-De La Cruz, ${ }^{90,{ }^{\text {hh }}}$ R. I. Rabadan-Trejo, ${ }^{90}$ R. Lopez-Fernandez, ${ }^{90}$ J. Mejia Guisao, ${ }^{90}$ R Reyes-Almanza, ${ }^{90}$ M. Ramirez-Garcia, ${ }^{90}$ A. Sanchez-Hernandez, ${ }^{90}$ S. Carrillo Moreno, ${ }^{91}$ C. Oropeza Barrera, ${ }^{91}$ F. Vazquez Valencia, ${ }^{91}$ J. Eysermans, ${ }^{92}$ I. Pedraza, ${ }^{92}$ H. A. Salazar Ibarguen, ${ }^{92}$ C. Uribe Estrada, ${ }^{92}$ A. Morelos Pineda, ${ }^{93}$ D. Krofcheck, ${ }^{94}$ S. Bheesette, ${ }^{95}$ P. H. Butler, ${ }^{95}$ A. Ahmad, ${ }^{96}$ M. Ahmad, ${ }^{96}$ M. I. Asghar, ${ }^{96}$ Q. Hassan, ${ }^{96}$ H. R. Hoorani, ${ }^{96}$ A. Saddique, ${ }^{96}$ M. A. Shah, ${ }^{96}$ M. Shoaib, ${ }^{96}$ M. Waqas, ${ }^{96}$ H. Bialkowska, ${ }^{97}$ M. Bluj, ${ }^{97}$ B. Boimska, ${ }^{97}$ T. Frueboes, ${ }^{97}$ M. Górski, ${ }^{97}$ M. Kazana, ${ }^{97}$ K. Nawrocki, ${ }^{97}$ M. Szleper, ${ }^{97}$ P. Traczyk, ${ }^{97}$ P. Zalewski, ${ }^{97}$ K. Bunkowski, ${ }^{98}$ A. Byszuk, ${ }^{98, i i}$ K. Doroba, ${ }^{98}$ A. Kalinowski, ${ }^{98}$ M. Konecki, ${ }^{98}$ J. Krolikowski, ${ }^{98}$ M. Misiura, ${ }^{98}$ M. Olszewski, ${ }^{98}$ A. Pyskir, ${ }^{98}$ M. Walczak, ${ }^{98}$ P. Bargassa, ${ }^{99}$ C. Beirão Da Cruz E Silva, ${ }^{99}$ A. Di Francesco, ${ }^{99}$ P. Faccioli, ${ }^{99}$ B. Galinhas, ${ }^{99}$ M. Gallinaro, ${ }^{99}$ J. Hollar, $^{99}$ N. Leonardo, ${ }^{99}$

L. Lloret Iglesias, ${ }^{99}$ M. V. Nemallapudi, ${ }^{99}$ J. Seixas, ${ }^{99}$ G. Strong, ${ }^{99}$ O. Toldaiev, ${ }^{99}$ D. Vadruccio, ${ }^{99}$ J. Varela, ${ }^{99}$

M. Gavrilenko, ${ }^{100}$ A. Golunov, ${ }^{100}$ I. Golutvin, ${ }^{100}$ N. Gorbounov, ${ }^{100}$ I. Gorbunov, ${ }^{100}$ A. Kamenev, ${ }^{100}$ V. Karjavin, ${ }^{100}$ V. Korenkov, ${ }^{100}$ A. Lanev, ${ }^{100}$ A. Malakhov, ${ }^{100}$ V. Matveev, ${ }^{100, j j, k k}$ P. Moisenz, ${ }^{100}$ V. Palichik, ${ }^{100}$ V. Perelygin, ${ }^{100}$ M. Savina, ${ }^{100}$ S. Shmatov, ${ }^{100}$ V. Smirnov, ${ }^{100}$ N. Voytishin, ${ }^{100}$ A. Zarubin, ${ }^{100}$ V. Golovtsov, ${ }^{101}$ Y. Ivanov, ${ }^{101}$ V. Kim, ${ }^{101,11}$

E. Kuznetsova, ${ }^{101, \mathrm{~mm}}$ P. Levchenko, ${ }^{101}$ V. Murzin, ${ }^{101}$ V. Oreshkin, ${ }^{101}$ I. Smirnov, ${ }^{101}$ D. Sosnov, ${ }^{101}$ V. Sulimov, ${ }^{101}$ L. Uvarov, ${ }^{101}$ S. Vavilov, ${ }^{101}$ A. Vorobyev, ${ }^{101}$ Yu. Andreev, ${ }^{102}$ A. Dermenev, ${ }^{102}$ S. Gninenko, ${ }^{102}$ N. Golubev, ${ }^{102}$

A. Karneyeu, ${ }^{102}$ M. Kirsanov, ${ }^{102}$ N. Krasnikov, ${ }^{102}$ A. Pashenkov, ${ }^{102}$ D. Tlisov, ${ }^{102}$ A. Toropin, ${ }^{102}$ V. Epshteyn, ${ }^{103}$ V. Gavrilov, ${ }^{103}$ N. Lychkovskaya, ${ }^{103}$ V. Popov, ${ }^{103}$ I. Pozdnyakov, ${ }^{103}$ G. Safronov, ${ }^{103}$ A. Spiridonov, ${ }^{103}$ A. Stepennov, ${ }^{103}$ V. Stolin, ${ }^{103}$ M. Toms, ${ }^{103}$ E. Vlasov, ${ }^{103}$ A. Zhokin, ${ }^{103}$ T. Aushev, ${ }^{104}$ M. Chadeeva, ${ }^{105, n n}$ P. Parygin, ${ }^{105}$ D. Philippov, ${ }^{105}$ S. Polikarpov, ${ }^{105, \mathrm{nn}}$ E. Popova, ${ }^{105}$ V. Rusinov, ${ }^{105}$ V. Andreev, ${ }^{106}$ M. Azarkin, ${ }^{106, k \mathrm{kk}}$ I. Dremin, ${ }^{106, k \mathrm{k}}$ M. Kirakosyan, ${ }^{106, \mathrm{kk}}$ S. V. Rusakov, ${ }^{106}$ A. Terkulov, ${ }^{106}$ A. Baskakov, ${ }^{107}$ A. Belyaev, ${ }^{107}$ E. Boos, ${ }^{107}$ M. Dubinin, ${ }^{107,00}$ L. Dudko, ${ }^{107}$ A. Ershov, ${ }^{107}$ A. Gribushin, ${ }^{107}$ V. Klyukhin, ${ }^{107}$ O. Kodolova, ${ }^{107}$ I. Lokhtin, ${ }^{107}$ I. Miagkov, ${ }^{107}$ S. Obraztsov, ${ }^{107}$ S. Petrushanko, ${ }^{107}$ V. Savrin, ${ }^{107}$ A. Snigirev, ${ }^{107}$ V. Blinov, ${ }^{108, p p}$ T. Dimova, ${ }^{108, p p}$ L. Kardapoltsev, ${ }^{108, p p}$ D. Shtol, ${ }^{108, p p}$ Y. Skovpen, ${ }^{108, p p}$ I. Azhgirey, ${ }^{109}$ I. Bayshev, ${ }^{109}$ S. Bitioukov, ${ }^{109}$ D. Elumakhov, ${ }^{109}$ A. Godizov, ${ }^{109}$ V. Kachanov, ${ }^{109}$ A. Kalinin, ${ }^{109}$ D. Konstantinov, ${ }^{109}$ P. Mandrik, ${ }^{109}$ V. Petrov, ${ }^{109}$ R. Ryutin, ${ }^{109}$ S. Slabospitskii, ${ }^{109}$ A. Sobol, ${ }^{109}$ S. Troshin, ${ }^{109}$ N. Tyurin, ${ }^{109}$ A. Uzunian, ${ }^{109}$ A. Volkov, ${ }^{109}$ A. Babaev, ${ }^{110}$ S. Baidali, ${ }^{110}$ V. Okhotnikov, ${ }^{110}$ P. Adzic, ${ }^{111, q q}$ P. Cirkovic, ${ }^{111}$ D. Devetak, ${ }^{111}$ M. Dordevic, ${ }^{111}$ J. Milosevic, ${ }^{111}$ J. Alcaraz Maestre, ${ }^{112}$ A. Álvarez Fernández, ${ }^{112}$ I. Bachiller, ${ }^{112}$ M. Barrio Luna, ${ }^{112}$ J. A. Brochero Cifuentes, ${ }^{112}$ M. Cerrada, ${ }^{112}$ N. Colino, ${ }^{112}$ B. De La Cruz, ${ }^{112}$ A. Delgado Peris, ${ }^{112}$ C. Fernandez Bedoya, ${ }^{112}$ J. P. Fernández Ramos, ${ }^{112}$ J. Flix, ${ }^{112}$ M. C. Fouz, ${ }^{112}$ O. Gonzalez Lopez, ${ }^{112}$ S. Goy Lopez, ${ }^{112}$ J. M. Hernandez, ${ }^{112}$ M. I. Josa, ${ }^{112}$ D. Moran, ${ }^{112}$ A. Pérez-Calero Yzquierdo, ${ }^{112}$ J. Puerta Pelayo, ${ }^{112}$ I. Redondo, ${ }^{112}$ L. Romero, ${ }^{112}$ M. S. Soares, ${ }^{112}$ A. Triossi, ${ }^{112}$ C. Albajar, ${ }^{113}$ J. F. de Trocóniz, ${ }^{113}$ J. Cuevas, ${ }^{114}$ C. Erice, ${ }^{114}$ J. Fernandez Menendez, ${ }^{114}$ S. Folgueras, ${ }^{114}$ I. Gonzalez Caballero, ${ }^{114}$ J. R. González Fernández, ${ }^{114}$ E. Palencia Cortezon, ${ }^{114}$ V. Rodríguez Bouza, ${ }^{114}$ S. Sanchez Cruz, ${ }^{114}$ 
P. Vischia, ${ }^{114}$ J. M. Vizan Garcia, ${ }^{114}$ I. J. Cabrillo, ${ }^{115}$ A. Calderon, ${ }^{115}$ B. Chazin Quero, ${ }^{115}$ J. Duarte Campderros, ${ }^{115}$ M. Fernandez, ${ }^{115}$ P. J. Fernández Manteca, ${ }^{115}$ A. García Alonso, ${ }^{115}$ J. Garcia-Ferrero, ${ }^{115}$ G. Gomez, ${ }^{115}$ A. Lopez Virto, ${ }^{115}$ J. Marco, ${ }^{115}$ C. Martinez Rivero, ${ }^{115}$ P. Martinez Ruiz del Arbol, ${ }^{115}$ F. Matorras, ${ }^{115}$ J. Piedra Gomez,${ }^{115}$ C. Prieels, ${ }^{115}$ T. Rodrigo, ${ }^{115}$ A. Ruiz-Jimeno, ${ }^{115}$ L. Scodellaro, ${ }^{115}$ N. Trevisani, ${ }^{115}$ I. Vila,${ }^{115}$ R. Vilar Cortabitarte, ${ }^{115}$ D. Abbaneo, ${ }^{116}$ B. Akgun, ${ }^{116}$ E. Auffray, ${ }^{16}$ P. Baillon, ${ }^{116}$ A. H. Ball, ${ }^{116}$ D. Barney, ${ }^{116}$ J. Bendavid, ${ }^{116}$ M. Bianco, ${ }^{116}$ A. Bocci, ${ }^{116}$ C. Botta, ${ }^{116}$ E. Brondolin, ${ }^{116}$ T. Camporesi, ${ }^{116}$ M. Cepeda,${ }^{116}$ G. Cerminara, ${ }^{116}$ E. Chapon, ${ }^{116}$ Y. Chen, ${ }^{116}$ G. Cucciati, ${ }^{116}$ D. d'Enterria, ${ }^{116}$ A. Dabrowski, ${ }^{116}$ V. Daponte, ${ }^{116}$ A. David, ${ }^{116}$ A. De Roeck, ${ }^{116}$ N. Deelen, ${ }^{116}$ M. Dobson, ${ }^{116}$ M. Dünser, ${ }^{116}$ N. Dupont, ${ }^{116}$

A. Elliott-Peisert, ${ }^{116}$ P. Everaerts, ${ }^{116}$ F. Fallavollita, ${ }^{16, \text { rr }}$ D. Fasanella, ${ }^{116}$ G. Franzoni, ${ }^{116}$ J. Fulcher, ${ }^{116}$ W. Funk, ${ }^{116}$ D. Gigi, ${ }^{116}$ A. Gilbert, ${ }^{116}$ K. Gill, ${ }^{116}$ F. Glege,${ }^{116}$ M. Guilbaud, ${ }^{116}$ D. Gulhan, ${ }^{116}$ J. Hegeman, ${ }^{116}$ V. Innocente, ${ }^{116}$ A. Jafari, ${ }^{116}$ P. Janot, ${ }^{116}$ O. Karacheban, ${ }^{116, t}$ J. Kieseler, ${ }^{116}$ A. Kornmayer, ${ }^{116}$ M. Krammer, ${ }^{116, b}$ C. Lange, ${ }^{116}$ P. Lecoq, ${ }^{116}$ C. Lourenço, ${ }^{116}$ L. Malgeri, ${ }^{116}$ M. Mannelli, ${ }^{116}$ F. Meijers, ${ }^{116}$ J. A. Merlin, ${ }^{116}$ S. Mersi, ${ }^{116}$ E. Meschi, ${ }^{116}$ P. Milenovic, ${ }^{116, s s}$ F. Moortgat, ${ }^{116}$ M. Mulders, ${ }^{116}$ J. Ngadiuba, ${ }^{116}$ S. Orfanelli, ${ }^{116}$ L. Orsini, ${ }^{116}$ F. Pantaleo, ${ }^{116, q}$ L. Pape, ${ }^{116}$ E. Perez, ${ }^{116}$ M. Peruzzi, ${ }^{116}$ A. Petrilli, ${ }^{116}$ G. Petrucciani, ${ }^{116}$ A. Pfeiffer, ${ }^{116}$ M. Pierini, ${ }^{116}$ F. M. Pitters, ${ }^{116}$ D. Rabady, ${ }^{116}$ A. Racz, ${ }^{116}$ T. Reis, ${ }^{116}$ G. Rolandi, ${ }^{16, t t}$ M. Rovere, ${ }^{116}$ H. Sakulin, ${ }^{116}$ C. Schäfer, ${ }^{116}$ C. Schwick, ${ }^{116}$ M. Seidel, ${ }^{116}$ M. Selvaggi, ${ }^{116}$ A. Sharma, ${ }^{116}$ P. Silva, ${ }^{116}$ P. Sphicas, ${ }^{11, \text {,uu }}$ A. Stakia, ${ }^{116}$ J. Steggemann, ${ }^{116}$ M. Tosi, ${ }^{116}$ D. Treille, ${ }^{116}$ A. Tsirou, ${ }^{116}$ V. Veckalns, ${ }^{116, v v}$ W. D. Zeuner, ${ }^{116}$ L. Caminada, ${ }^{117, \text { ww }}$ K. Deiters, ${ }^{117}$ W. Erdmann, ${ }^{117}$ R. Horisberger, ${ }^{117}$ Q. Ingram, ${ }^{117}$ H. C. Kaestli, ${ }^{117}$

D. Kotlinski, ${ }^{117}$ U. Langenegger, ${ }^{117}$ T. Rohe, ${ }^{117}$ S. A. Wiederkehr, ${ }^{117}$ M. Backhaus,${ }^{118}$ L. Bäni, ${ }^{118}$ P. Berger,${ }^{118}$ N. Chernyavskaya, ${ }^{118}$ G. Dissertori, ${ }^{118}$ M. Dittmar, ${ }^{118}$ M. Donegà, ${ }^{118}$ C. Dorfer, ${ }^{118}$ C. Grab, ${ }^{118}$ C. Heidegger, ${ }^{18}$ D. Hits, ${ }^{118}$ J. Hoss, ${ }^{118}$ T. Klijnsma, ${ }^{118}$ W. Lustermann, ${ }^{118}$ R. A. Manzoni, ${ }^{118}$ M. Marionneau, ${ }^{118}$ M. T. Meinhard ${ }^{118}$ F. Micheli, ${ }^{118}$ P. Musella, ${ }^{118}$ F. Nessi-Tedaldi, ${ }^{118}$ J. Pata, ${ }^{118}$ F. Pauss, ${ }^{118}$ G. Perrin, ${ }^{118}$ L. Perrozzi, ${ }^{118}$ S. Pigazzini, ${ }^{118}$ M. Quittnat, ${ }^{118}$ D. Ruini, ${ }^{118}$ D. A. Sanz Becerra, ${ }^{118}$ M. Schönenberger, ${ }^{118}$ L. Shchutska, ${ }^{118}$ V. R. Tavolaro,${ }^{118}$ K. Theofilatos, ${ }^{118}$

M. L. Vesterbacka Olsson, ${ }^{118}$ R. Wallny, ${ }^{118}$ D. H. Zhu, ${ }^{118}$ T. K. Aarrestad, ${ }^{119}$ C. Amsler, ${ }^{19, x x}$ D. Brzhechko, ${ }^{119}$ M. F. Canelli, ${ }^{119}$ A. De Cosa, ${ }^{119}$ R. Del Burgo, ${ }^{119}$ S. Donato, ${ }^{119}$ C. Galloni,${ }^{119}$ T. Hreus, ${ }^{119}$ B. Kilminster, ${ }^{119}$ I. Neutelings, ${ }^{119}$ D. Pinna, ${ }^{119}$ G. Rauco, ${ }^{119}$ P. Robmann, ${ }^{119}$ D. Salerno, ${ }^{119}$ K. Schweiger, ${ }^{119}$ C. Seitz ${ }^{119}$ Y. Takahashi, ${ }^{119}$ A. Zucchetta, ${ }^{119}$ Y. H. Chang, ${ }^{120}$ K. y. Cheng, ${ }^{120}$ T. H. Doan, ${ }^{120}$ Sh. Jain, ${ }^{120}$ R. Khurana, ${ }^{120}$ C. M. Kuo, ${ }^{120}$ W. Lin, ${ }^{120}$ A. Pozdnyakov, ${ }^{120}$ S. S. Yu, ${ }^{120}$ P. Chang, ${ }^{121}$ Y. Chao, ${ }^{121}$ K. F. Chen, ${ }^{121}$ P. H. Chen, ${ }^{121}$ W.-S. Hou, ${ }^{121}$ Arun Kumar, ${ }^{121}$ Y. y. Li, ${ }^{121}$ Y. F. Liu, ${ }^{121}$

R.-S. Lu, ${ }^{121}$ E. Paganis, ${ }^{121}$ A. Psallidas, ${ }^{121}$ A. Steen, ${ }^{121}$ J.f. Tsai, ${ }^{121}$ B. Asavapibhop, ${ }^{122}$ N. Srimanobhas, ${ }^{122}$

N. Suwonjandee, ${ }^{122}$ A. Bat, ${ }^{123}$ F. Boran, ${ }^{123}$ S. Cerci, ${ }^{123, y y}$ S. Damarseckin, ${ }^{123}$ Z. S. Demiroglu, ${ }^{123}$ F. Dolek, ${ }^{123}$ C. Dozen, ${ }^{123}$ I. Dumanoglu, ${ }^{123}$ S. Girgis, ${ }^{123}$ G. Gokbulut, ${ }^{123}$ Y. Guler, ${ }^{123}$ E. Gurpinar, ${ }^{123}$ I. Hos, ${ }^{123, z z}$ C. Isik, ${ }^{123}$ E. E. Kangal, ${ }^{123, \text { aaa }}$ O. Kara, ${ }^{123}$ A. Kayis Topaksu, ${ }^{123}$ U. Kiminsu, ${ }^{123}$ M. Oglakci, ${ }^{123}$ G. Onengut, ${ }^{123}$ K. Ozdemir, ${ }^{123, \text { bb }}$ S. Ozturk, ${ }^{123, c c c}$ D. Sunar Cerci, ${ }^{123, y y}$ B. Tali, ${ }^{123, y y}$ U. G. Tok, ${ }^{123}$ S. Turkcapar, ${ }^{123}$ I. S. Zorbakir, ${ }^{123}$ C. Zorbilmez, ${ }^{123}$ B. Isildak, ${ }^{124, d d d}$ G. Karapinar, ${ }^{124, \text { eee }}$ M. Yalvac, ${ }^{124}$ M. Zeyrek, ${ }^{124}$ I. O. Atakisi, ${ }^{125}$ E. Gülmez, ${ }^{125}$ M. Kaya, ${ }^{125, f f f}$ O. Kaya, ${ }^{125, \text { ggg }}$ S. Tekten, ${ }^{125}$ E. A. Yetkin, ${ }^{125, \text { hhh }}$ M. N. Agaras, ${ }^{126}$ S. Atay, ${ }^{126}$ A. Cakir, ${ }^{126}$ K. Cankocak, ${ }^{126}$ Y. Komurcu, ${ }^{126}$ S. Sen, ${ }^{126, i i i}$ B. Grynyov, ${ }^{127}$ L. Levchuk, ${ }^{128}$ F. Ball, ${ }^{129}$ L. Beck, ${ }^{129}$ J. J. Brooke, ${ }^{129}$ D. Burns, ${ }^{129}$ E. Clement, ${ }^{129}$ D. Cussans, ${ }^{129}$ O. Davignon, ${ }^{129}$ H. Flacher, ${ }^{129}$ J. Goldstein, ${ }^{129}$ G. P. Heath, ${ }^{129}$ H. F. Heath, ${ }^{129}$ L. Kreczko, ${ }^{129}$ D. M. Newbold, ${ }^{129, j j j}$ S. Paramesvaran, ${ }^{129}$ B. Penning, ${ }^{129}$ T. Sakuma, ${ }^{129}$ D. Smith, ${ }^{129}$ V. J. Smith, ${ }^{129}$ J. Taylor, ${ }^{12}$ A. Titterton, ${ }^{129}$ K. W. Bell, ${ }^{130}$ A. Belyaev, ${ }^{13, k k k}$ C. Brew, ${ }^{130}$ R. M. Brown, ${ }^{130}$ D. Cieri, ${ }^{130}$ D. J. A. Cockerill, ${ }^{130}$ J. A. Coughlan, ${ }^{130}$ K. Harder, ${ }^{130}$ S. Harper, ${ }^{130}$ J. Linacre, ${ }^{130}$ E. Olaiya, ${ }^{130}$ D. Petyt, ${ }^{130}$ C. H. Shepherd-Themistocleous, ${ }^{130}$ A. Thea, ${ }^{130}$ I. R. Tomalin, ${ }^{130}$ T. Williams, ${ }^{130}$

W. J. Womersley, ${ }^{130}$ G. Auzinger, ${ }^{131}$ R. Bainbridge, ${ }^{131}$ P. Bloch, ${ }^{131}$ J. Borg, ${ }^{131}$ S. Breeze, ${ }^{131}$ O. Buchmuller, ${ }^{131}$ A. Bundock, ${ }^{131}$ S. Casasso, ${ }^{131}$ D. Colling, ${ }^{131}$ L. Corpe,${ }^{131}$ P. Dauncey,${ }^{131}$ G. Davies, ${ }^{131}$ M. Della Negra ${ }^{131}$ R. Di Maria, ${ }^{131}$ Y. Haddad ${ }^{131}$ G. Hall, ${ }^{131}$ G. Iles, ${ }^{131}$ T. James, ${ }^{131}$ M. Komm, ${ }^{131}$ C. Laner, ${ }^{131}$ L. Lyons, ${ }^{131}$ A.-M. Magnan, ${ }^{131}$ S. Malik, ${ }^{131}$ A. Martelli, ${ }^{131}$ J. Nash, ${ }^{131,111}$ A. Nikitenko, ${ }^{131, \mathrm{~h}}$ V. Palladino, ${ }^{131}$ M. Pesaresi, ${ }^{131}$ A. Richards, ${ }^{131}$ A. Rose, ${ }^{131}$ E. Scott, ${ }^{131}$ C. Seez ${ }^{131}$ A. Shtipliyski, ${ }^{131}$ G. Singh, ${ }^{131}$ M. Stoye, ${ }^{131}$ T. Strebler,${ }^{131}$ S. Summers, ${ }^{131}$ A. Tapper, ${ }^{131}$ K. Uchida, ${ }^{131}$ T. Virdee, ${ }^{131, q}$ N. Wardle, ${ }^{131}$ D. Winterbottom, ${ }^{131}$ J. Wright, ${ }^{131}$ S. C. Zenz, ${ }^{131}$ J. E. Cole, ${ }^{132}$ P. R. Hobson, ${ }^{132}$ A. Khan, ${ }^{132}$ P. Kyberd, ${ }^{132}$ C. K. Mackay, ${ }^{132}$ A. Morton, ${ }^{132}$ I. D. Reid ${ }^{132}$ L. Teodorescu, ${ }^{132}$ S. Zahid, ${ }^{132}$ K. Call, ${ }^{133}$ J. Dittmann, ${ }^{133}$ K. Hatakeyama, ${ }^{133}$ H. Liu, ${ }^{133}$ C. Madrid, ${ }^{133}$ B. Mcmaster, ${ }^{133}$ N. Pastika, ${ }^{133}$ C. Smith, ${ }^{133}$ R. Bartek, ${ }^{134}$ A. Dominguez, ${ }^{134}$ A. Buccilli, ${ }^{135}$ S. I. Cooper, ${ }^{135}$ C. Henderson, ${ }^{135}$ P. Rumerio, ${ }^{135}$ C. West,${ }^{135}$ D. Arcaro, ${ }^{136}$ T. Bose, ${ }^{136}$ D. Gastler, ${ }^{136}$ D. Rankin, ${ }^{136}$ C. Richardson, ${ }^{136}$ J. Rohlf, ${ }^{136}$ L. Sulak, ${ }^{136}$ D. Zou, ${ }^{136}$ G. Benelli, ${ }^{137}$ X. Coubez, ${ }^{137}$ D. Cutts, ${ }^{137}$ M. Hadley, ${ }^{137}$ J. Hakala, ${ }^{137}$ U. Heintz, ${ }^{137}$ J. M. Hogan, ${ }^{137, \mathrm{mmm}}$ K. H. M. Kwok, ${ }^{137}$ E. Laird, ${ }^{137}$ G. Landsberg, ${ }^{137}$ J. Lee, ${ }^{137}$ Z. Mao, ${ }^{137}$ 
M. Narain, ${ }^{137}$ S. Piperov, ${ }^{137}$ S. Sagir, ${ }^{137, n n n}$ R. Syarif, ${ }^{137}$ E. Usai, ${ }^{137}$ D. Yu, ${ }^{137}$ R. Band, ${ }^{138}$ C. Brainerd, ${ }^{138}$ R. Breedon, ${ }^{138}$ D. Burns, ${ }^{138}$ M. Calderon De La Barca Sanchez, ${ }^{138}$ M. Chertok, ${ }^{138}$ J. Conway, ${ }^{138}$ R. Conway, ${ }^{138}$ P. T. Cox, ${ }^{138}$ R. Erbacher, ${ }^{138}$ C. Flores, ${ }^{138}$ G. Funk, ${ }^{138}$ W. Ko, ${ }^{138}$ O. Kukral, ${ }^{138}$ R. Lander, ${ }^{138}$ C. Mclean, ${ }^{138}$ M. Mulhearn, ${ }^{138}$ D. Pellett, ${ }^{138}$ J. Pilot, ${ }^{138}$ S. Shalhout, ${ }^{138}$ M. Shi, ${ }^{138}$ D. Stolp, ${ }^{138}$ D. Taylor, ${ }^{138}$ K. Tos, ${ }^{138}$ M. Tripathi, ${ }^{138}$ Z. Wang, ${ }^{138}$ F. Zhang, ${ }^{138}$ M. Bachtis, ${ }^{139}$ C. Bravo, ${ }^{139}$ R. Cousins, ${ }^{139}$ A. Dasgupta, ${ }^{139}$ A. Florent, ${ }^{139}$ J. Hauser, ${ }^{139}$ M. Ignatenko, ${ }^{139}$ N. Mccoll, ${ }^{139}$ S. Regnard, ${ }^{139}$ D. Saltzberg, ${ }^{139}$ C. Schnaible, ${ }^{139}$ V. Valuev, ${ }^{139}$ E. Bouvier, ${ }^{140}$ K. Burt, ${ }^{140}$ R. Clare, ${ }^{140}$ J. W. Gary, ${ }^{140}$

S. M. A. Ghiasi Shirazi, ${ }^{140}$ G. Hanson, ${ }^{140}$ G. Karapostoli, ${ }^{140}$ E. Kennedy, ${ }^{140}$ F. Lacroix, ${ }^{140}$ O. R. Long, ${ }^{140}$ M. Olmedo Negrete, ${ }^{140}$ M. I. Paneva, ${ }^{140}$ W. Si, ${ }^{140}$ L. Wang, ${ }^{140}$ H. Wei, ${ }^{140}$ S. Wimpenny, ${ }^{140}$ B. R. Yates, ${ }^{140}$ J. G. Branson, ${ }^{141}$ S. Cittolin, ${ }^{141}$ M. Derdzinski, ${ }^{141}$ R. Gerosa, ${ }^{141}$ D. Gilbert, ${ }^{141}$ B. Hashemi, ${ }^{141}$ A. Holzner, ${ }^{141}$ D. Klein, ${ }^{141}$ G. Kole, ${ }^{141}$ V. Krutelyov, ${ }^{141}$ J. Letts, ${ }^{141}$ M. Masciovecchio, ${ }^{141}$ D. Olivito, ${ }^{141}$ S. Padhi, ${ }^{141}$ M. Pieri, ${ }^{141}$ M. Sani, ${ }^{141}$ V. Sharma, ${ }^{141}$ S. Simon, ${ }^{141}$ M. Tadel, ${ }^{141}$ A. Vartak, ${ }^{141}$ S. Wasserbaech, ${ }^{141,00 o}$ J. Wood, ${ }^{141}$ F. Würthwein, ${ }^{141}$ A. Yagil, ${ }^{141}$

G. Zevi Della Porta, ${ }^{141}$ N. Amin, ${ }^{142}$ R. Bhandari, ${ }^{142}$ J. Bradmiller-Feld, ${ }^{142}$ C. Campagnari, ${ }^{142}$ M. Citron, ${ }^{142}$ A. Dishaw, ${ }^{142}$ V. Dutta, ${ }^{142}$ M. Franco Sevilla, ${ }^{142}$ L. Gouskos, ${ }^{142}$ R. Heller, ${ }^{142}$ J. Incandela, ${ }^{142}$ A. Ovcharova, ${ }^{142}$ H. Qu, ${ }^{142}$ J. Richman, ${ }^{142}$ D. Stuart, ${ }^{142}$ I. Suarez, ${ }^{142}$ S. Wang, ${ }^{142}$ J. Yoo, ${ }^{142}$ D. Anderson, ${ }^{143}$ A. Bornheim, ${ }^{143}$ J. M. Lawhorn, ${ }^{143}$ H. B. Newman, ${ }^{143}$ T. Q. Nguyen, ${ }^{143}$ M. Spiropulu, ${ }^{143}$ J. R. Vlimant, ${ }^{143}$ R. Wilkinson, ${ }^{143}$ S. Xie, ${ }^{143}$ Z. Zhang, ${ }^{143}$ R. Y. Zhu, ${ }^{143}$ M. B. Andrews, ${ }^{144}$ T. Ferguson, ${ }^{144}$ T. Mudholkar, ${ }^{144}$ M. Paulini, ${ }^{144}$ M. Sun, ${ }^{144}$ I. Vorobiev, ${ }^{144}$ M. Weinberg, ${ }^{144}$ J. P. Cumalat, ${ }^{145}$ W. T. Ford, ${ }^{145}$

F. Jensen, ${ }^{145}$ A. Johnson, ${ }^{145}$ M. Krohn, ${ }^{145}$ S. Leontsinis, ${ }^{145}$ E. MacDonald, ${ }^{145}$ T. Mulholland, ${ }^{145}$ K. Stenson, ${ }^{145}$ K. A. Ulmer, ${ }^{145}$ S. R. Wagner, ${ }^{145}$ J. Alexander, ${ }^{146}$ J. Chaves, ${ }^{146}$ Y. Cheng, ${ }^{146}$ J. Chu, ${ }^{146}$ A. Datta, ${ }^{146}$ K. Mcdermott, ${ }^{146}$ N. Mirman, ${ }^{146}$ J. R. Patterson, ${ }^{146}$ D. Quach, ${ }^{146}$ A. Rinkevicius, ${ }^{146}$ A. Ryd, ${ }^{146}$ L. Skinnari, ${ }^{146}$ L. Soffi, ${ }^{146}$ S. M. Tan, ${ }^{146}$ Z. Tao, ${ }^{146}$ J. Thom, ${ }^{146}$ J. Tucker, ${ }^{146}$ P. Wittich, ${ }^{146}$ M. Zientek, ${ }^{146}$ S. Abdullin, ${ }^{147}$ M. Albrow, ${ }^{147}$ M. Alyari, ${ }^{147}$ G. Apollinari, ${ }^{147}$ A. Apresyan, ${ }^{147}$ A. Apyan, ${ }^{147}$ S. Banerjee, ${ }^{147}$ L. A. T. Bauerdick, ${ }^{147}$ A. Beretvas, ${ }^{147}$ J. Berryhill, ${ }^{147}$ P. C. Bhat, ${ }^{147}$ G. Bolla, ${ }^{147, a}$ K. Burkett, ${ }^{147}$ J. N. Butler, ${ }^{147}$ A. Canepa, ${ }^{147}$ G. B. Cerati, ${ }^{147}$ H. W. K. Cheung, ${ }^{147}$ F. Chlebana, ${ }^{147}$ M. Cremonesi, ${ }^{147}$ J. Duarte, ${ }^{147}$ V. D. Elvira, ${ }^{147}$ J. Freeman, ${ }^{147}$ Z. Gecse, ${ }^{147}$ E. Gottschalk, ${ }^{147}$ L. Gray, ${ }^{147}$ D. Green, ${ }^{147}$ S. Grünendahl, ${ }^{147}$ O. Gutsche, ${ }^{147}$ J. Hanlon, ${ }^{147}$ R. M. Harris, ${ }^{147}$ S. Hasegawa, ${ }^{147}$ J. Hirschauer, ${ }^{147}$ Z. Hu, ${ }^{147}$ B. Jayatilaka, ${ }^{147}$ S. Jindariani, ${ }^{147}$ M. Johnson, ${ }^{147}$ U. Joshi, ${ }^{147}$ B. Klima, ${ }^{147}$ M. J. Kortelainen, ${ }^{147}$ B. Kreis, ${ }^{147}$ S. Lammel, ${ }^{147}$ D. Lincoln, ${ }^{147}$ R. Lipton, ${ }^{147}$ M. Liu, ${ }^{147}$ T. Liu, ${ }^{147}$ J. Lykken, ${ }^{147}$ K. Maeshima, ${ }^{147}$ J. M. Marraffino, ${ }^{147}$ D. Mason, ${ }^{147}$ P. McBride, ${ }^{147}$ P. Merkel, ${ }^{147}$ S. Mrenna, ${ }^{147}$ S. Nahn, ${ }^{147}$ V. O’Dell, ${ }^{147}$ K. Pedro, ${ }^{147}$ C. Pena, ${ }^{147}$ O. Prokofyev, ${ }^{147}$ G. Rakness, ${ }^{147}$ L. Ristori, ${ }^{147}$ A. Savoy-Navarro, ${ }^{147, p p p}$ B. Schneider, ${ }^{147}$ E. Sexton-Kennedy, ${ }^{147}$ A. Soha, ${ }^{147}$ W. J. Spalding, ${ }^{147}$ L. Spiegel, ${ }^{147}$ S. Stoynev, ${ }^{147}$ J. Strait, ${ }^{147}$ N. Strobbe, ${ }^{147}$ L. Taylor, ${ }^{147}$ S. Tkaczyk, ${ }^{147}$ N. V. Tran, ${ }^{147}$ L. Uplegger, ${ }^{147}$ E. W. Vaandering, ${ }^{147}$ C. Vernieri, ${ }^{147}$ M. Verzocchi, ${ }^{147}$ R. Vidal, ${ }^{147}$ M. Wang, ${ }^{147}$ H. A. Weber, ${ }^{147}$ A. Whitbeck, ${ }^{147}$ D. Acosta, ${ }^{148}$ P. Avery, ${ }^{148}$ P. Bortignon, ${ }^{148}$ D. Bourilkov, ${ }^{148}$ A. Brinkerhoff, ${ }^{148}$ L. Cadamuro, ${ }^{148}$ A. Carnes, ${ }^{148}$ M. Carver, ${ }^{148}$ D. Curry, ${ }^{148}$ R. D. Field, ${ }^{148}$ S. V. Gleyzer, ${ }^{148}$ B. M. Joshi, ${ }^{148}$ J. Konigsberg, ${ }^{148}$ A. Korytov, ${ }^{148}$ P. Ma, ${ }^{148}$ K. Matchev, ${ }^{148}$ H. Mei, ${ }^{148}$ G. Mitselmakher, ${ }^{148}$ K. Shi, ${ }^{148}$ D. Sperka, ${ }^{148}$ J. Wang, ${ }^{148}$ S. Wang, ${ }^{148}$ Y. R. Joshi, ${ }^{149}$ S. Linn, ${ }^{149}$ A. Ackert, ${ }^{150}$ T. Adams, ${ }^{150}$ A. Askew, ${ }^{150}$ S. Hagopian, ${ }^{150}$ V. Hagopian, ${ }^{150}$ K. F. Johnson, ${ }^{150}$ T. Kolberg, ${ }^{150}$ G. Martinez, ${ }^{150}$ T. Perry, ${ }^{150}$ H. Prosper, ${ }^{150}$ A. Saha, ${ }^{150}$ V. Sharma, ${ }^{150}$ R. Yohay, ${ }^{150}$ M. M. Baarmand, ${ }^{151}$ V. Bhopatkar, ${ }^{151}$ S. Colafranceschi, ${ }^{151}$ M. Hohlmann, ${ }^{151}$ D. Noonan, ${ }^{151}$ M. Rahmani, ${ }^{151}$ T. Roy, ${ }^{151}$ F. Yumiceva, ${ }^{151}$ M. R. Adams, ${ }^{152}$ L. Apanasevich, ${ }^{152}$ D. Berry, ${ }^{152}$ R. R. Betts, ${ }^{152}$ R. Cavanaugh, ${ }^{152}$ X. Chen, ${ }^{152}$ S. Dittmer, ${ }^{152}$ O. Evdokimov, ${ }^{152}$ C. E. Gerber, ${ }^{152}$ D. A. Hangal, ${ }^{152}$ D. J. Hofman, ${ }^{152}$ K. Jung, ${ }^{152}$ J. Kamin, ${ }^{152}$ C. Mills, ${ }^{152}$

I. D. Sandoval Gonzalez, ${ }^{152}$ M. B. Tonjes, ${ }^{152}$ N. Varelas, ${ }^{152}$ H. Wang, ${ }^{152}$ X. Wang, ${ }^{152}$ Z. Wu, ${ }^{152}$ J. Zhang, ${ }^{152}$

M. Alhusseini, ${ }^{153}$ B. Bilki, ${ }^{153, q q q}$ W. Clarida, ${ }^{153}$ K. Dilsiz, ${ }^{153, \text { rrr }}$ S. Durgut, ${ }^{153}$ R. P. Gandrajula, ${ }^{153}$ M. Haytmyradov, ${ }^{153}$ V. Khristenko, ${ }^{153}$ J.-P. Merlo, ${ }^{153}$ A. Mestvirishvili, ${ }^{153}$ A. Moeller, ${ }^{153}$ J. Nachtman, ${ }^{153}$ H. Ogul, ${ }^{153, \text { sss }}$ Y. Onel, ${ }^{153}$ F. Ozok, ${ }^{153, t t t}$ A. Penzo, ${ }^{153}$ C. Snyder, ${ }^{153}$ E. Tiras, ${ }^{153}$ J. Wetzel, ${ }^{153}$ B. Blumenfeld, ${ }^{154}$ A. Cocoros, ${ }^{154}$ N. Eminizer, ${ }^{154}$ D. Fehling, ${ }^{154}$ L. Feng, ${ }^{154}$ A. V. Gritsan, ${ }^{154}$ W. T. Hung, ${ }^{154}$ P. Maksimovic,${ }^{154}$ J. Roskes, ${ }^{154}$ U. Sarica, ${ }^{154}$ M. Swartz, ${ }^{154}$ M. Xiao, ${ }^{154}$ C. You, ${ }^{154}$ A. Al-bataineh, ${ }^{155}$ P. Baringer, ${ }^{155}$ A. Bean, ${ }^{155}$ S. Boren, ${ }^{155}$ J. Bowen, ${ }^{155}$ A. Bylinkin, ${ }^{155}$ J. Castle, ${ }^{155}$ S. Khalil, ${ }^{155}$ A. Kropivnitskaya, ${ }^{155}$ D. Majumder, ${ }^{155}$ W. Mcbrayer, ${ }^{155}$ M. Murray, ${ }^{155}$ C. Rogan, ${ }^{155}$ S. Sanders, ${ }^{155}$ E. Schmitz, ${ }^{155}$ J. D. Tapia Takaki, ${ }^{155}$ Q. Wang, ${ }^{155}$ S. Duric, ${ }^{156}$ A. Ivanov, ${ }^{156}$ K. Kaadze, ${ }^{156}$ D. Kim, ${ }^{156}$ Y. Maravin, ${ }^{156}$ D. R. Mendis, ${ }^{156}$ T. Mitchell, ${ }^{156}$ A. Modak, ${ }^{156}$ A. Mohammadi, ${ }^{156}$ L. K. Saini, ${ }^{156}$ N. Skhirtladze, ${ }^{156}$ F. Rebassoo, ${ }^{157}$ D. Wright, ${ }^{157}$ A. Baden, ${ }^{158}$ O. Baron, ${ }^{158}$ A. Belloni, ${ }^{158}$ S. C. Eno, ${ }^{158}$ Y. Feng, ${ }^{158}$ C. Ferraioli, ${ }^{158}$ N. J. Hadley, ${ }^{158}$ S. Jabeen, ${ }^{158}$ G. Y. Jeng, ${ }^{158}$ R. G. Kellogg, ${ }^{158}$ J. Kunkle, ${ }^{158}$ A. C. Mignerey, ${ }^{158}$ F. Ricci-Tam, ${ }^{158}$ Y. H. Shin, ${ }^{158}$ A. Skuja, ${ }^{158}$ S. C. Tonwar, ${ }^{158}$ K. Wong, ${ }^{158}$ D. Abercrombie, ${ }^{159}$ B. Allen, ${ }^{159}$ V. Azzolini, ${ }^{159}$ A. Baty, ${ }^{159}$ G. Bauer, ${ }^{159}$ R. Bi, ${ }^{159}$ S. Brandt, ${ }^{159}$ W. Busza, ${ }^{159}$ I. A. Cali, ${ }^{159}$ 
M. D’ Alfonso, ${ }^{159}$ Z. Demiragli, ${ }^{159}$ G. Gomez Ceballos, ${ }^{159}$ M. Goncharov, ${ }^{159}$ P. Harris, ${ }^{159}$ D. Hsu, ${ }^{159}$ M. Hu, ${ }^{159}$ Y. liyama, ${ }^{159}$ G. M. Innocenti, ${ }^{159}$ M. Klute, ${ }^{159}$ D. Kovalskyi, ${ }^{159}$ Y.-J. Lee, ${ }^{159}$ P. D. Luckey, ${ }^{159}$ B. Maier, ${ }^{159}$ A. C. Marini,${ }^{159}$ C. Mcginn, ${ }^{159}$ C. Mironov, ${ }^{159}$ S. Narayanan, ${ }^{159}$ X. Niu, ${ }^{159}$ C. Paus, ${ }^{159}$ C. Roland, ${ }^{159}$ G. Roland, ${ }^{159}$ G. S. F. Stephans, ${ }^{159}$ K. Sumorok, ${ }^{159}$ K. Tatar, ${ }^{159}$ D. Velicanu, ${ }^{159}$ J. Wang, ${ }^{159}$ T. W. Wang, ${ }^{159}$ B. Wyslouch, ${ }^{159}$ S. Zhaozhong, ${ }^{159}$ A. C. Benvenuti, ${ }^{160}$ R. M. Chatterjee, ${ }^{160}$ A. Evans, ${ }^{160}$ P. Hansen, ${ }^{160}$ S. Kalafut, ${ }^{160}$ Y. Kubota, ${ }^{160}$ Z. Lesko, ${ }^{160}$ J. Mans, ${ }^{160}$ S. Nourbakhsh, ${ }^{160}$ N. Ruckstuhl, ${ }^{160}$ R. Rusack, ${ }^{160}$ J. Turkewitz, ${ }^{160}$ M. A. Wadud, ${ }^{160}$ J. G. Acosta, ${ }^{161}$ S. Oliveros, ${ }^{161}$ E. Avdeeva, ${ }^{162}$ K. Bloom, ${ }^{162}$ D. R. Claes, ${ }^{162}$ C. Fangmeier, ${ }^{162}$ F. Golf, ${ }^{162}$ R. Gonzalez Suarez, ${ }^{162}$ R. Kamalieddin, ${ }^{162}$ I. Kravchenko, ${ }^{162}$ J. Monroy, ${ }^{162}$ J. E. Siado, ${ }^{162}$ G. R. Snow, ${ }^{162}$ B. Stieger, ${ }^{162}$ A. Godshalk, ${ }^{163}$ C. Harrington, ${ }^{163}$ I. Iashvili, ${ }^{163}$ A. Kharchilava, ${ }^{163}$ D. Nguyen, ${ }^{163}$ A. Parker, ${ }^{163}$ S. Rappoccio, ${ }^{163}$ B. Roozbahani, ${ }^{163}$ G. Alverson, ${ }^{164}$ E. Barberis, ${ }^{164}$ C. Freer, ${ }^{164}$ A. Hortiangtham, ${ }^{164}$ D. M. Morse, ${ }^{164}$ T. Orimoto, ${ }^{164}$ R. Teixeira De Lima, ${ }^{164}$ T. Wamorkar, ${ }^{164}$ B. Wang, ${ }^{164}$ A. Wisecarver, ${ }^{164}$ D. Wood, ${ }^{164}$ S. Bhattacharya, ${ }^{165}$ O. Charaf, ${ }^{165}$ K. A. Hahn, ${ }^{165}$ N. Mucia, ${ }^{165}$ N. Odell, ${ }^{165}$ M. H. Schmitt, ${ }^{165}$ K. Sung, ${ }^{165}$ M. Trovato, ${ }^{165}$ M. Velasco, ${ }^{165}$ R. Bucci, ${ }^{166}$ N. Dev, ${ }^{166}$ M. Hildreth ${ }^{166}$ K. Hurtado Anampa, ${ }^{166}$ C. Jessop, ${ }^{166}$ D. J. Karmgard, ${ }^{166}$ N. Kellams, ${ }^{166}$ K. Lannon, ${ }^{166}$ W. Li, ${ }^{166}$ N. Loukas, ${ }^{166}$ N. Marinelli, ${ }^{166}$ F. Meng, ${ }^{166}$ C. Mueller, ${ }^{166}$ Y. Musienko, ${ }^{166, j \mathrm{jj}}$ M. Planer, ${ }^{166}$ A. Reinsvold, ${ }^{166}$ R. Ruchti, ${ }^{166}$ P. Siddireddy, ${ }^{166}$ G. Smith, ${ }^{166}$ S. Taroni, ${ }^{166}$ M. Wayne, ${ }^{166}$ A. Wightman, ${ }^{166}$ M. Wolf, ${ }^{166}$ A. Woodard ${ }^{166}$ J. Alimena, ${ }^{167}$ L. Antonelli, ${ }^{167}$ B. Bylsma, ${ }^{167}$ L. S. Durkin, ${ }^{167}$ S. Flowers,${ }^{167}$ B. Francis, ${ }^{167}$ A. Hart, ${ }^{167}$ C. Hill, ${ }^{167}$ W. Ji, ${ }^{167}$ T. Y. Ling, ${ }^{167}$ W. Luo, ${ }^{167}$ B. L. Winer, ${ }^{167}$ H. W. Wulsin, ${ }^{167}$ S. Cooperstein, ${ }^{168}$ P. Elmer, ${ }^{168}$ J. Hardenbrook, ${ }^{168}$ P. Hebda, ${ }^{168}$ S. Higginbotham, ${ }^{168}$ A. Kalogeropoulos, ${ }^{168}$ D. Lange, ${ }^{168}$ M. T. Lucchini, ${ }^{168}$ J. Luo, ${ }^{168}$ D. Marlow, ${ }^{168}$ K. Mei, ${ }^{168}$ I. Ojalvo, ${ }^{168}$ J. Olsen, ${ }^{168}$ C. Palmer, ${ }^{168}$ P. Piroué, ${ }^{168}$ J. Salfeld-Nebgen, ${ }^{168}$ D. Stickland, ${ }^{168}$ C. Tully, ${ }^{168}$ S. Malik, ${ }^{169}$ S. Norberg, ${ }^{169}$ A. Barker ${ }^{170}$ V. E. Barnes, ${ }^{170}$ S. Das, ${ }^{170}$ L. Gutay, ${ }^{170}$ M. Jones, ${ }^{170}$ A. W. Jung, ${ }^{170}$ A. Khatiwada, ${ }^{170}$ B. Mahakud, ${ }^{170}$ D. H. Miller, ${ }^{170}$ N. Neumeister, ${ }^{170}$ C. C. Peng, ${ }^{170}$ H. Qiu, ${ }^{170}$ J. F. Schulte,${ }^{170}$ J. Sun, ${ }^{170}$ F. Wang, ${ }^{170}$ R. Xiao, ${ }^{170}$ W. Xie, ${ }^{170}$ T. Cheng, ${ }^{171}$ J. Dolen, ${ }^{171}$ N. Parashar,${ }^{171}$ Z. Chen, ${ }^{172}$ K. M. Ecklund, ${ }^{172}$ S. Freed, ${ }^{172}$ F. J. M. Geurts, ${ }^{172}$ M. Kilpatrick, ${ }^{172}$ W. Li, ${ }^{172}$ B. Michlin, ${ }^{172}$ B. P. Padley, ${ }^{172}$ J. Roberts, ${ }^{172}$ J. Rorie, ${ }^{172}$ W. Shi, ${ }^{172}$ Z. Tu, ${ }^{172}$ J. Zabel, ${ }^{172}$ A. Zhang, ${ }^{172}$ A. Bodek, ${ }^{173}$ P. de Barbaro, ${ }^{173}$ R. Demina, ${ }^{173}$ Y.t. Duh, ${ }^{173}$ J. L. Dulemba, ${ }^{173}$ C. Fallon, ${ }^{173}$ T. Ferbel, ${ }^{173}$ M. Galanti, ${ }^{173}$ A. Garcia-Bellido, ${ }^{173}$ J. Han,${ }^{173}$ O. Hindrichs, ${ }^{173}$ A. Khukhunaishvili, ${ }^{173}$ K. H. Lo,${ }^{173}$ P. Tan, ${ }^{173}$ R. Taus, ${ }^{173}$ M. Verzetti, ${ }^{173}$ A. Agapitos, ${ }^{174}$ J. P. Chou, ${ }^{174}$ Y. Gershtein, ${ }^{174}$ T. A. Gómez Espinosa, ${ }^{174}$ E. Halkiadakis, ${ }^{174}$ M. Heindl, ${ }^{174}$ E. Hughes, ${ }^{174}$ S. Kaplan, ${ }^{174}$ R. Kunnawalkam Elayavalli, ${ }^{174}$ S. Kyriacou, ${ }^{174}$ A. Lath, ${ }^{174}$ R. Montalvo, ${ }^{174}$ K. Nash, ${ }^{174}$ M. Osherson, ${ }^{174}$ H. Saka, ${ }^{174}$ S. Salur, ${ }^{174}$ S. Schnetzer, ${ }^{174}$ D. Sheffield, ${ }^{174}$ S. Somalwar, ${ }^{174}$ R. Stone, ${ }^{174}$ S. Thomas, ${ }^{174}$ P. Thomassen, ${ }^{174}$ M. Walker, ${ }^{174}$ A. G. Delannoy, ${ }^{175}$ J. Heideman, ${ }^{175}$ G. Riley, ${ }^{175}$ S. Spanier, ${ }^{175}$ K. Thapa, ${ }^{175}$ O. Bouhali, ${ }^{176, \text { uuu }}$ A. Celik, ${ }^{176}$ M. Dalchenko, ${ }^{176}$ M. De Mattia, ${ }^{176}$ A. Delgado, ${ }^{176}$ S. Dildick, ${ }^{176}$ R. Eusebi, ${ }^{176}$ J. Gilmore, ${ }^{176}$ T. Huang, ${ }^{176}$ T. Kamon, ${ }^{176, v v v}$ S. Luo, ${ }^{176}$ R. Mueller, ${ }^{176}$ R. Patel,${ }^{176}$ A. Perloff, ${ }^{176}$ L. Perniè, ${ }^{176}$ D. Rathjens, ${ }^{176}$ A. Safonov, ${ }^{176}$ N. Akchurin, ${ }^{177}$ J. Damgov, ${ }^{177}$ F. De Guio, ${ }^{177}$ P. R. Dudero, ${ }^{177}$ S. Kunori,,${ }^{177}$ K. Lamichhane, ${ }^{177}$ S. W. Lee, ${ }^{177}$ T. Mengke, ${ }^{177}$ S. Muthumuni, ${ }^{177}$ T. Peltola, ${ }^{177}$ S. Undleeb, ${ }^{177}$ I. Volobouev, ${ }^{177}$ Z. Wang, ${ }^{177}$ S. Greene, ${ }^{178}$ A. Gurrola, ${ }^{178}$ R. Janjam, ${ }^{178}$

W. Johns, ${ }^{178}$ C. Maguire, ${ }^{178}$ A. Melo, ${ }^{178} \mathrm{H}$. Ni, ${ }^{178}$ K. Padeken, ${ }^{178}$ J. D. Ruiz Alvarez, ${ }^{178}$ P. Sheldon, ${ }^{178}$ S. Tuo, ${ }^{178}$

J. Velkovska, ${ }^{178}$ M. Verweij, ${ }^{178}$ Q. Xu,${ }^{178}$ M. W. Arenton, ${ }^{179}$ P. Barria, ${ }^{179}$ B. Cox ${ }^{179}$ R. Hirosky, ${ }^{179}$ M. Joyce, ${ }^{179}$

A. Ledovskoy, ${ }^{179}$ H. Li, ${ }^{179}$ C. Neu,${ }^{179}$ T. Sinthuprasith, ${ }^{179}$ Y. Wang, ${ }^{179}$ E. Wolfe, ${ }^{179}$ F. Xia, ${ }^{179}$ R. Harr, ${ }^{180}$ P. E. Karchin, ${ }^{180}$ N. Poudyal, ${ }^{180}$ J. Sturdy, ${ }^{180}$ P. Thapa ${ }^{180}$ S. Zaleski, ${ }^{180}$ M. Brodski, ${ }^{181}$ J. Buchanan, ${ }^{181}$ C. Caillol,${ }^{181}$ D. Carlsmith,${ }^{181}$ S. Dasu, ${ }^{181}$ L. Dodd ${ }^{181}$ B. Gomber ${ }^{181}$ M. Grothe, ${ }^{181}$ M. Herndon, ${ }^{181}$ A. Hervé, ${ }^{181}$ U. Hussain, ${ }^{181}$ P. Klabbers ${ }^{181}$ A. Lanaro, ${ }^{181}$ A. Levine, ${ }^{181}$ K. Long, ${ }^{181}$ R. Loveless, ${ }^{181}$ T. Ruggles, ${ }^{181}$ A. Savin, ${ }^{181}$ N. Smith, ${ }^{181}$ W. H. Smith, ${ }^{181}$ and N. Woods ${ }^{181}$

\title{
(CMS Collaboration)
}

\author{
${ }^{1}$ Yerevan Physics Institute, Yerevan, Armenia \\ ${ }^{2}$ Institut für Hochenergiephysik, Wien, Austria \\ ${ }^{3}$ Institute for Nuclear Problems, Minsk, Belarus \\ ${ }^{4}$ Universiteit Antwerpen, Antwerpen, Belgium \\ ${ }^{5}$ Vrije Universiteit Brussel, Brussel, Belgium \\ ${ }^{6}$ Université Libre de Bruxelles, Bruxelles, Belgium \\ ${ }^{7}$ Ghent University, Ghent, Belgium \\ ${ }^{8}$ Université Catholique de Louvain, Louvain-la-Neuve, Belgium \\ ${ }^{9}$ Centro Brasileiro de Pesquisas Fisicas, Rio de Janeiro, Brazil
}


${ }^{10}$ Universidade do Estado do Rio de Janeiro, Rio de Janeiro, Brazil

${ }^{11 a}$ Universidade Estadual Paulista, São Paulo, Brazil

${ }^{11 \mathrm{~b}}$ Universidade Federal do ABC, São Paulo, Brazil

${ }^{12}$ Institute for Nuclear Research and Nuclear Energy, Bulgarian Academy of Sciences, Sofia, Bulgaria

${ }^{13}$ University of Sofia, Sofia, Bulgaria

${ }^{14}$ Beihang University, Beijing, China

${ }^{15}$ Institute of High Energy Physics, Beijing, China

${ }^{16}$ State Key Laboratory of Nuclear Physics and Technology, Peking University, Beijing, China

${ }^{17}$ Tsinghua University, Beijing, China

${ }^{18}$ Universidad de Los Andes, Bogota, Colombia

${ }^{19}$ University of Split, Faculty of Electrical Engineering, Mechanical Engineering and Naval Architecture, Split, Croatia

${ }^{20}$ University of Split, Faculty of Science, Split, Croatia

${ }^{21}$ Institute Rudjer Boskovic, Zagreb, Croatia

${ }^{22}$ University of Cyprus, Nicosia, Cyprus

${ }^{23}$ Charles University, Prague, Czech Republic

${ }^{24}$ Escuela Politecnica Nacional, Quito, Ecuador

${ }^{25}$ Universidad San Francisco de Quito, Quito, Ecuador

${ }^{26}$ Academy of Scientific Research and Technology of the Arab Republic of Egypt,

Egyptian Network of High Energy Physics, Cairo, Egypt

${ }^{27}$ National Institute of Chemical Physics and Biophysics, Tallinn, Estonia

${ }^{28}$ Department of Physics, University of Helsinki, Helsinki, Finland

${ }^{29}$ Helsinki Institute of Physics, Helsinki, Finland

${ }^{30}$ Lappeenranta University of Technology, Lappeenranta, Finland

${ }^{31}$ IRFU, CEA, Université Paris-Saclay, Gif-sur-Yvette, France

${ }^{32}$ Laboratoire Leprince-Ringuet, Ecole polytechnique, CNRS/IN2P3, Université Paris-Saclay, Palaiseau, France

${ }^{33}$ Université de Strasbourg, CNRS, IPHC UMR 7178, F-67000 Strasbourg, France

${ }^{34}$ Centre de Calcul de l'Institut National de Physique Nucleaire et de Physique des Particules, CNRS/IN2P3, Villeurbanne, France

${ }^{35}$ Université de Lyon, Université Claude Bernard Lyon 1, CNRS-IN2P3,

Institut de Physique Nucléaire de Lyon, Villeurbanne, France

${ }^{36}$ Georgian Technical University, Tbilisi, Georgia

${ }^{37}$ Tbilisi State University, Tbilisi, Georgia

${ }^{38}$ RWTH Aachen University, I. Physikalisches Institut, Aachen, Germany

${ }^{39}$ RWTH Aachen University, III. Physikalisches Institut A, Aachen, Germany

${ }^{40}$ RWTH Aachen University, III. Physikalisches Institut B, Aachen, Germany

${ }^{41}$ Deutsches Elektronen-Synchrotron, Hamburg, Germany

${ }^{42}$ University of Hamburg, Hamburg, Germany

${ }^{43}$ Karlsruher Institut fuer Technology, Karlsruhe, Germany

${ }^{44}$ Institute of Nuclear and Particle Physics (INPP), NCSR Demokritos, Aghia Paraskevi, Greece

${ }^{45}$ National and Kapodistrian University of Athens, Athens, Greece

${ }^{46}$ National Technical University of Athens, Athens, Greece

${ }^{47}$ University of Ioánnina, Ioánnina, Greece

${ }^{48}$ MTA-ELTE Lendület CMS Particle and Nuclear Physics Group, Eötvös Loránd University, Budapest, Hungary

${ }^{49}$ Wigner Research Centre for Physics, Budapest, Hungary

${ }^{50}$ Institute of Nuclear Research ATOMKI, Debrecen, Hungary

${ }^{51}$ Institute of Physics, University of Debrecen, Debrecen, Hungary

${ }^{52}$ Indian Institute of Science (IISc), Bangalore, India

${ }^{53}$ National Institute of Science Education and Research, HBNI, Bhubaneswar, India

${ }^{54}$ Panjab University, Chandigarh, India

${ }^{55}$ University of Delhi, Delhi, India

${ }^{56}$ Saha Institute of Nuclear Physics, HBNI, Kolkata,India

${ }^{57}$ Indian Institute of Technology Madras, Madras, India

${ }^{58}$ Bhabha Atomic Research Centre, Mumbai, India

${ }^{59}$ Tata Institute of Fundamental Research-A, Mumbai, India

${ }^{60}$ Tata Institute of Fundamental Research-B, Mumbai, India

${ }^{61}$ Indian Institute of Science Education and Research (IISER), Pune, India

${ }^{62}$ Institute for Research in Fundamental Sciences (IPM), Tehran, Iran 
${ }^{63}$ University College Dublin, Dublin, Ireland

${ }^{64 a}$ INFN Sezione di Bari, Bari, Italy

${ }^{64 \mathrm{~b}}$ Università di Bari, Bari, Italy

${ }^{64 \mathrm{c}}$ Politecnico di Bari, Bari, Italy

${ }^{65 a}$ INFN Sezione di Bologna, Bologna, Italy

${ }^{65 \mathrm{~b}}$ Università di Bologna, Bologna, Italy

${ }^{66 \mathrm{a}}$ INFN Sezione di Catania, Catania, Italy

${ }^{66 \mathrm{~b}}$ Università di Catania, Catania, Italy

${ }^{67 \mathrm{a}}$ INFN Sezione di Firenze, Firenze, Italy

${ }^{67 \mathrm{~b}}$ Università di Firenze, Firenze, Italy

${ }^{68}$ INFN Laboratori Nazionali di Frascati, Frascati, Italy

${ }^{69 a}$ INFN Sezione di Genova, Genova, Italy

${ }^{69 \mathrm{~b}}$ Università di Genova, Genova, Italy

${ }^{70 a}$ INFN Sezione di Milano-Bicocca, Milano, Italy

${ }^{70 \mathrm{~b}}$ Università di Milano-Bicocca, Milano, Italy

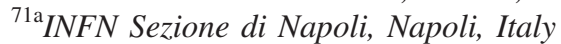

${ }^{71 b}$ Università di Napoli 'Federico II', Napoli, Italy

${ }^{71 c}$ Università della Basilicata, Potenza, Italy

${ }^{71 d}$ Università G. Marconi, Roma, Italy

${ }^{72 a}$ INFN Sezione di Padova, Padova, Italy

${ }^{72 \mathrm{~b}}$ Università di Padova, Padova, Italy

${ }^{72 \mathrm{c}}$ Università di Trento, Trento, Italy

${ }^{73 a}$ INFN Sezione di Pavia, Pavia, Italy

${ }^{73 b}$ Università di Pavia, Pavia, Italy

${ }^{74 a}$ INFN Sezione di Perugia, Perugia, Italy

${ }^{74 \mathrm{~b}}$ Università di Perugia, Perugia, Italy

${ }^{75 a}$ INFN Sezione di Pisa, Pisa, Italy

${ }^{75 b}$ Università di Pisa, Pisa, Italy

${ }^{75 c}$ Scuola Normale Superiore di Pisa, Pisa, Italy

${ }^{76 a}$ INFN Sezione di Roma, Rome, Italy

${ }^{76 \mathrm{~b}}$ Sapienza Università di Roma, Rome, Italy

${ }^{77 a}$ INFN Sezione di Torino, Torino, Italy

${ }^{77 b}$ Università di Torino, Torino, Italy

${ }^{77 \mathrm{c}}$ Università del Piemonte Orientale, Novara, Italy

${ }^{78 \mathrm{a}}$ INFN Sezione di Trieste, Trieste, Italy

${ }^{78 b}$ Università di Trieste, Trieste, Italy

${ }^{79}$ Kyungpook National University, Daegu, Korea

${ }^{80}$ Chonnam National University, Institute for Universe and Elementary Particles, Kwangju, Korea

${ }^{81}$ Hanyang University, Seoul, Korea

${ }^{82}$ Korea University, Seoul, Korea

${ }^{83}$ Sejong University, Seoul, Korea

${ }^{84}$ Seoul National University, Seoul, Korea

${ }^{85}$ University of Seoul, Seoul, Korea

${ }^{86}$ Sungkyunkwan University, Suwon, Korea

${ }^{87}$ Vilnius University, Vilnius, Lithuania

${ }^{88}$ National Centre for Particle Physics, Universiti Malaya, Kuala Lumpur, Malaysia

${ }^{89}$ Universidad de Sonora (UNISON), Hermosillo, Mexico

${ }^{90}$ Centro de Investigacion y de Estudios Avanzados del IPN, Mexico City, Mexico

${ }^{91}$ Universidad Iberoamericana, Mexico City, Mexico

${ }^{92}$ Benemerita Universidad Autonoma de Puebla, Puebla, Mexico

${ }^{93}$ Universidad Autónoma de San Luis Potosí, San Luis Potosí, Mexico

${ }^{94}$ University of Auckland, Auckland, New Zealand

${ }^{95}$ University of Canterbury, Christchurch, New Zealand

${ }^{96}$ National Centre for Physics, Quaid-I-Azam University, Islamabad, Pakistan

${ }^{97}$ National Centre for Nuclear Research, Swierk, Poland

${ }^{98}$ Institute of Experimental Physics, Faculty of Physics, University of Warsaw, Warsaw, Poland

${ }^{99}$ Laboratório de Instrumentação e Física Experimental de Partículas, Lisboa, Portugal

${ }^{100}$ Joint Institute for Nuclear Research, Dubna, Russia

${ }^{101}$ Petersburg Nuclear Physics Institute, Gatchina (St. Petersburg), Russia

${ }^{102}$ Institute for Nuclear Research, Moscow, Russia 
${ }^{103}$ Institute for Theoretical and Experimental Physics, Moscow, Russia

${ }^{104}$ Moscow Institute of Physics and Technology, Moscow, Russia

${ }^{105}$ National Research Nuclear University 'Moscow Engineering Physics Institute' (MEPhI),

Moscow, Russia

${ }^{106}$ P.N. Lebedev Physical Institute, Moscow, Russia

${ }^{107}$ Skobeltsyn Institute of Nuclear Physics, Lomonosov Moscow State University, Moscow, Russia

${ }^{108}$ Novosibirsk State University (NSU), Novosibirsk, Russia

${ }^{109}$ State Research Center of Russian Federation, Institute for High Energy Physics of NRC 'Kurchatov Institute', Protvino, Russia

${ }^{110}$ National Research Tomsk Polytechnic University, Tomsk, Russia

${ }^{111}$ University of Belgrade, Faculty of Physics and Vinca Institute of Nuclear Sciences, Belgrade, Serbia

${ }^{112}$ Centro de Investigaciones Energéticas Medioambientales y Tecnológicas (CIEMAT), Madrid, Spain

${ }^{113}$ Universidad Autónoma de Madrid, Madrid, Spain

${ }^{114}$ Universidad de Oviedo, Oviedo, Spain

${ }^{115}$ Instituto de Física de Cantabria (IFCA), CSIC-Universidad de Cantabria, Santander, Spain

${ }^{116}$ CERN, European Organization for Nuclear Research, Geneva, Switzerland

${ }^{117}$ Paul Scherrer Institut, Villigen, Switzerland

${ }^{118}$ ETH Zurich - Institute for Particle Physics and Astrophysics (IPA), Zurich, Switzerland

${ }^{119}$ Universität Zürich, Zurich, Switzerland

${ }^{120}$ National Central University, Chung-Li, Taiwan

${ }^{121}$ National Taiwan University (NTU), Taipei, Taiwan

${ }^{122}$ Chulalongkorn University, Faculty of Science, Department of Physics, Bangkok, Thailand

${ }^{123}$ Cukurova University, Physics Department, Science and Art Faculty, Adana, Turkey

${ }^{124}$ Middle East Technical University, Physics Department, Ankara, Turkey

${ }^{125}$ Bogazici University, Istanbul, Turkey

${ }^{126}$ Istanbul Technical University, Istanbul, Turkey

${ }^{127}$ Institute for Scintillation Materials of National Academy of Science of Ukraine, Kharkov, Ukraine

${ }^{128}$ National Scientific Center, Kharkov Institute of Physics and Technology, Kharkov, Ukraine

${ }^{129}$ University of Bristol, Bristol, United Kingdom

${ }^{130}$ Rutherford Appleton Laboratory, Didcot, United Kingdom

${ }^{131}$ Imperial College, London, United Kingdom

${ }^{132}$ Brunel University, Uxbridge, United Kingdom

${ }^{133}$ Baylor University, Waco, USA

${ }^{134}$ Catholic University of America, Washington DC, USA

${ }^{135}$ The University of Alabama, Tuscaloosa, USA

${ }^{136}$ Boston University, Boston, USA

${ }^{137}$ Brown University, Providence, USA

${ }^{138}$ University of California, Davis, Davis, USA

${ }^{139}$ University of California, Los Angeles, USA

${ }^{140}$ University of California, Riverside, Riverside, USA

${ }^{141}$ University of California, San Diego, La Jolla, USA

${ }^{142}$ University of California, Santa Barbara - Department of Physics, Santa Barbara, USA

${ }^{143}$ California Institute of Technology, Pasadena, USA

${ }^{144}$ Carnegie Mellon University, Pittsburgh, USA

${ }^{145}$ University of Colorado Boulder, Boulder, USA

${ }^{146}$ Cornell University, Ithaca, USA

${ }^{147}$ Fermi National Accelerator Laboratory, Batavia, USA

${ }^{148}$ University of Florida, Gainesville, USA

${ }^{149}$ Florida International University, Miami, USA

${ }^{150}$ Florida State University, Tallahassee, USA

${ }^{151}$ Florida Institute of Technology, Melbourne, USA

${ }^{152}$ University of Illinois at Chicago (UIC), Chicago, USA

${ }^{153}$ The University of Iowa, Iowa City, USA

${ }^{154}$ Johns Hopkins University, Baltimore, USA

${ }^{155}$ The University of Kansas, Lawrence, USA

${ }^{156}$ Kansas State University, Manhattan, USA

${ }^{157}$ Lawrence Livermore National Laboratory, Livermore, USA

${ }^{158}$ University of Maryland, College Park, USA

${ }^{159}$ Massachusetts Institute of Technology, Cambridge, USA

${ }^{160}$ University of Minnesota, Minneapolis, USA 


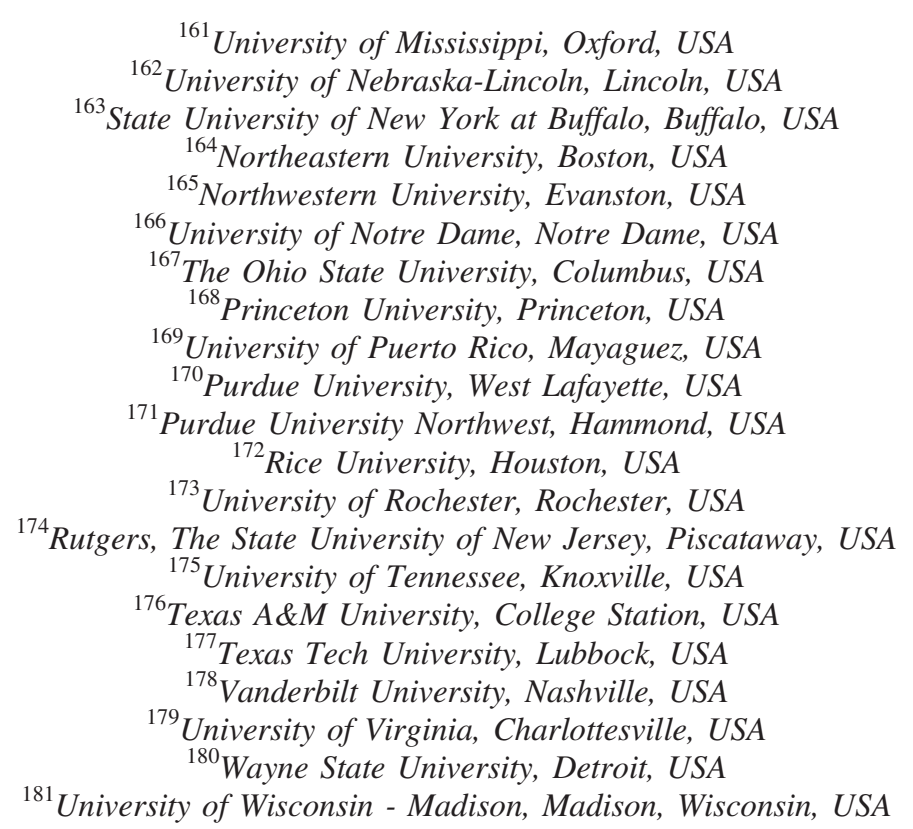

${ }^{\mathrm{a}}$ Deceased.

${ }^{\mathrm{b}}$ Also at Vienna University of Technology, Vienna, Austria.

${ }^{\mathrm{c}}$ Also at IRFU, CEA, Université Paris-Saclay, Gif-sur-Yvette, France.

${ }^{\mathrm{d}}$ Also at Universidade Estadual de Campinas, Campinas, Brazil.

${ }^{\mathrm{e}}$ Also at Federal University of Rio Grande do Sul, Porto Alegre, Brazil.

${ }^{\mathrm{f}}$ Also at Université Libre de Bruxelles, Bruxelles, Belgium.

${ }^{\mathrm{g}}$ Also at University of Chinese Academy of Sciences.

${ }^{\mathrm{h}}$ Also at Institute for Theoretical and Experimental Physics, Moscow, Russia.

${ }^{\mathrm{i}}$ Also at Joint Institute for Nuclear Research, Dubna, Russia.

${ }^{\mathrm{j}}$ Also at British University in Egypt, Cairo, Egypt.

${ }^{\mathrm{k}}$ Also at Suez University, Suez, Egypt.

${ }^{1}$ Also at Zewail City of Science and Technology, Zewail, Egypt.

${ }^{\mathrm{m}}$ Also at Helwan University, Cairo, Egypt.

${ }^{\mathrm{n}}$ Also at Department of Physics, King Abdulaziz University, Jeddah, Saudi Arabia.

${ }^{\circ}$ Also at Université de Haute Alsace, Mulhouse, France.

${ }^{\mathrm{p}}$ Also at Skobeltsyn Institute of Nuclear Physics, Lomonosov Moscow State University, Moscow, Russia.

${ }^{\mathrm{q}}$ Also at CERN, European Organization for Nuclear Research, Geneva, Switzerland.

${ }^{\mathrm{r}}$ Also at RWTH Aachen University, III. Physikalisches Institut A, Aachen, Germany.

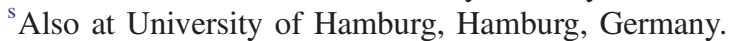

${ }^{\mathrm{t}}$ Also at Brandenburg University of Technology, Cottbus, Germany.

"Also at MTA-ELTE Lendület CMS Particle and Nuclear Physics Group, Eötvös Loránd University, Budapest, Hungary.

${ }^{v}$ Also at Institute of Nuclear Research ATOMKI, Debrecen, Hungary.

${ }^{\mathrm{w}}$ Also at Institute of Physics, University of Debrecen, Debrecen, Hungary.

${ }^{\mathrm{x}}$ Also at IIT Bhubaneswar, Bhubaneswar, India.

${ }^{y}$ Also at Institute of Physics, Bhubaneswar, India.

${ }^{\mathrm{z}}$ Also at Shoolini University, Solan, India.

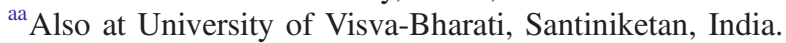

${ }^{\mathrm{bb}}$ Also at Isfahan University of Technology, Isfahan, Iran.

${ }^{c c}$ Also at Plasma Physics Research Center, Science and Research Branch, Islamic Azad University, Tehran, Iran.

${ }^{\mathrm{dd}}$ Also at Università degli Studi di Siena, Siena, Italy.

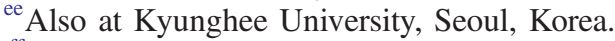

${ }^{\mathrm{ff}}$ Also at International Islamic University of Malaysia, Kuala Lumpur, Malaysia.

gg Also at Malaysian Nuclear Agency, MOSTI, Kajang, Malaysia.

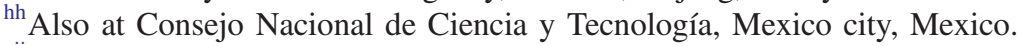

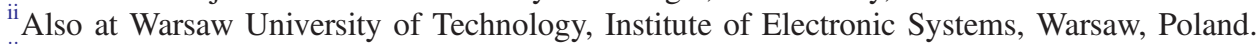

${ }^{\mathrm{jj}}$ Also at Institute for Nuclear Research, Moscow, Russia.

${ }^{\mathrm{kk}}$ Also at National Research Nuclear University 'Moscow Engineering Physics Institute' (MEPhI), Moscow, Russia.

${ }^{11}$ Also at St. Petersburg State Polytechnical University, St. Petersburg, Russia. 
${ }^{\mathrm{mm}}$ Also at University of Florida, Gainesville, USA.

${ }^{n n}$ Also at P.N. Lebedev Physical Institute, Moscow, Russia.

${ }^{\text {oo }}$ Also at California Institute of Technology, Pasadena, USA.

${ }^{\mathrm{pp}}$ Also at Budker Institute of Nuclear Physics, Novosibirsk, Russia.

${ }^{\mathrm{qq}}$ Also at Faculty of Physics, University of Belgrade, Belgrade, Serbia.

${ }^{\mathrm{rr}}$ Also at INFN Sezione di Pavia, Università di Pavia, Pavia, Italy.

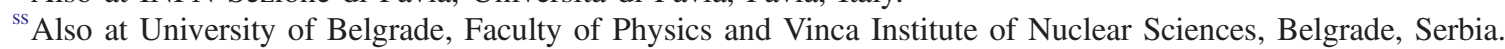

${ }^{\text {tt} A l s o ~ a t ~ S c u o l a ~ N o r m a l e ~ e ~ S e z i o n e ~ d e l l ' I N F N, ~ P i s a, ~ I t a l y . ~}$

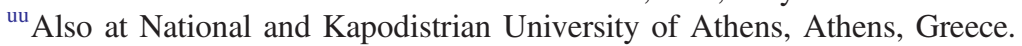

${ }^{\mathrm{vv}}$ Also at Riga Technical University, Riga, Latvia.

${ }^{\text {ww }}$ Also at Universität Zürich, Zurich, Switzerland.

${ }^{\mathrm{xx}}$ Also at Stefan Meyer Institute for Subatomic Physics, Vienna, Austria.

${ }^{\mathrm{yy}}$ Also at Adiyaman University, Adiyaman, Turkey.

${ }^{\mathrm{zz}}$ Also at Istanbul Aydin University, Istanbul, Turkey.

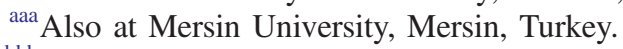

${ }^{b b b}$ Also at Piri Reis University, Istanbul, Turkey.

${ }^{c c c}$ Also at Gaziosmanpasa University, Tokat, Turkey.

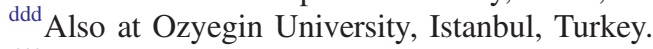

${ }^{\text {eee }}$ Also at Izmir Institute of Technology, Izmir, Turkey.

${ }^{\mathrm{fff}}$ Also at Marmara University, Istanbul, Turkey.

${ }^{\text {ggg }}$ Also at Kafkas University, Kars, Turkey.

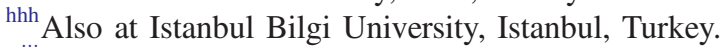

iii Also at Hacettepe University, Ankara, Turkey.

${ }_{\mathrm{jjj}}$ Also at Rutherford Appleton Laboratory, Didcot, United Kingdom.

${ }^{k k k}$ Also at School of Physics and Astronomy, University of Southampton, Southampton, United Kingdom.

${ }^{111}$ Also at Monash University, Faculty of Science, Clayton, Australia.

${ }^{\mathrm{mmm}}$ Also at Bethel University, Arden Hills, Minnesota, USA.

${ }^{n n n}$ Also at Karamanoğlu Mehmetbey University, Karaman, Turkey.

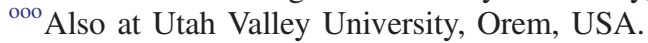

${ }^{\mathrm{ppp}}$ Also at Purdue University, West Lafayette, USA.

${ }^{\mathrm{qqq}}$ Also at Beykent University, Istanbul, Turkey.

${ }^{\mathrm{rrr}}$ Also at Bingol University, Bingol, Turkey.

${ }^{\text {sss }}$ Also at Sinop University, Sinop, Turkey.

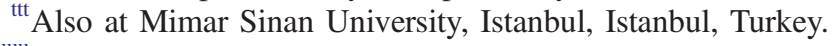

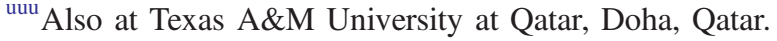

${ }^{\mathrm{vvv}}$ Also at Kyungpook National University, Daegu, Korea. 